\title{
Thermodynamic semirings
}

\author{
Matilde Marcolli and Ryan Thorngren
}

\begin{abstract}
The Witt construction describes a functor from the category of Rings to the category of characteristic 0 rings. It is uniquely determined by a few associativity constraints which do not depend on the types of the variables considered, in other words, by integer polynomials. This universality allowed Alain Connes and Caterina Consani to devise an analogue of the Witt ring for characteristic one, an attractive endeavour since we know very little about the arithmetic in this exotic characteristic and its corresponding field with one element. Interestingly, they found that in characteristic one, the Witt construction depends critically on the Shannon entropy. In the current work, we examine this surprising occurrence, defining a Witt operad for an arbitrary information measure and a corresponding algebra we call a thermodynamic semiring. This object exhibits algebraically many of the familiar properties of information measures, and we examine in particular the Tsallis and Renyi entropy functions and applications to nonextensive thermodynamics and multifractals. We find that the arithmetic of the thermodynamic semiring is exactly that of a certain guessing game played using the given information measure.
\end{abstract}

Mathematics Subject Classification (2010). 94A17, 13F35, 28D20.

Keywords. Entropy (Shannon, Renyi, Tsallis, Kullback-Leibler divergence), semiring, Witt construction, multifractals, operads, binary guessing games, entropy algebras.

\section{Contents}

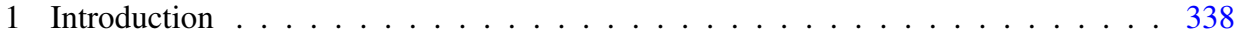

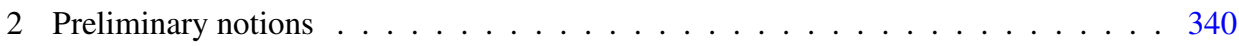

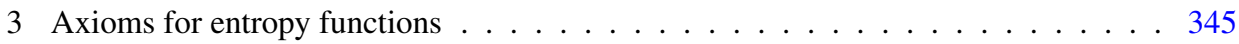

4 Thermodynamic semirings . . . . . . . . . . . . . . . . 348

5 Statistical mechanics . . . . . . . . . . . . . . . . . 351

6 The Rényi entropy . . . . . . . . . . . . . . . . . . . . . . . . . . 353

7 The Tsallis entropy . . . . . . . . . . . . . . . . . 355

8 The Kullback-Leibler divergence . . . . . . . . . . . . . . . . . . . . . . . 357

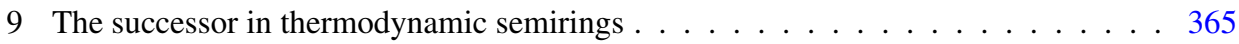

10 Entropy operad . . . . . . . . . . . . . . . . 370

11 Further perspectives and directions $\ldots \ldots \ldots$. . . . . . . . . 385

References . . . . . . . . . . . . . . . . . . . . . . . . . . 389 


\section{Introduction}

The past few years have seen several interesting new results focusing on various aspects of the elusive "geometry over the field with one element", see for instance [7] [11] [12] [32] [36], [48], among many others. The idea of $\mathbb{F}_{1}$-geometry has its roots in an observation of Tits [51] that limits as $q \rightarrow 1$ of counting functions for certain varieties defined over finite fields $\mathbb{F}_{q}$ exhibit an interesting combinatorial meaning, suggesting that the resulting combinatorial geometry should be seen as an algebraic geometry over a non-existent "field with one element" $\mathbb{F}_{1}$. Part of the motivation for developing a sufficiently refined theory of varieties and schemes over $\mathbb{F}_{1}$ lies in the idea that being able to cast $\operatorname{Spec} \mathbb{Z}$ in the role of a curve over a suitably defined Spec $\mathbb{F}_{1}$ may lead to finding an analog for number fields of the Weil proof [55] of the Riemann hypothesis for finite fields.

Among the existing approaches aimed at developing various aspects of geometry over $\mathbb{F}_{1}$, the one that is of direct interest to us in the present paper is a recent construction by Connes and Consani [10], [11] of semirings of characteristic one (a nilpotent hypothesis). These are endowed with an additive structure that provides an analog of the Witt formula for the addition of the multiplicative Teichmüller lifts in strict $p$-rings. As observed in [10] and [11], the commutativity, identity, and associativity conditions for this addition force the function used in defining the Witt sums in characteristic one to be equal to the Shannon entropy.

The goal of this paper is to explore this occurrence of the Shannon entropy in the characteristic one Witt construction of [10] and [11]. In particular, we show here that the construction introduced in those papers can be seen as part of a more general theory of "thermodynamic semirings", which encodes various properties of suitable "entropy functions" in terms of algebraic properties of the corresponding semirings.

After reviewing the case of [10], [11] in §2, we present a general definition and some basic properties of thermodynamic semirings in $\S 3$ and $\S 4$, based on the axiomatization of information-theoretic entropy through the Khinchin axioms and other equivalent formulations. We then give in $\$ 5$ a physical interpretation of the structure of thermodynamic semiring in terms of Statistical Mechanics, distinguishing between the extensive and non-extensive cases and the cases of ergodic and non-ergodic statistical systems. We see that the lack of associativity of the thermodynamic semiring has a natural physical interpretation in terms of mixing, chemical potentials, and free energy. This generalizes the thermodynamic interpretation of certain formulas from tropical mathematics considered in [43].

We focus then on specific examples of other important information-theoretic entropy functions, such as the Rényi entropy, the Tsallis entropy, or the Kullback-Leibler divergence, and we analyze in detail the properties of the corresponding thermodynamic semirings. In $\S 6$, we consider the case of the Rényi entropy, which is a one-parameter generalization of the Shannon entropy that still satisfies the extensivity property. In $\S 7$ we focus instead on the Tsallis entropy, which is a non-extensive one-parameter generalization of the Shannon entropy, and we show that a simple one- 
parameter deformation of the Witt construction of [10] and [11] identifies the Tsallis entropy as the unique information measure that satisfies the associativity constraint.

In $\S 8$ we consider the case of the Kullback-Leibler divergence or relative entropy (information gain), and we show that thermodynamic semirings based on this information measure can be associated to univariate and multivariate binary statistical manifolds, in the sense of information geometry, and to multifractal systems, in such a way that the algebraic properties of the semirings detect the statistical and multifractal properties of the underlying spaces. We also relate a hyperfield structure arising from the KL divergence to those considered in [54].

We also show in $\S 9$ that the algebraic structure of the thermodynamic semirings can be encoded in a suitably defined successor function and that the properties of this function and its iterates as a dynamical system capture both the algebraic structure of the semiring and the thermodynamical properties of the corresponding entropy measure. We give explicit examples of these successor functions and their behavior for the Shannon, Rényi, and Tsallis entropies. In $§ 9.3$ we show that this function has an interpretation as the cumulant generating function for the energy, which reveals some further thermodynamic details of our construction.

Finally, in $\S 10$, we phrase our construction using operads whose composition trees suggest an interpretation in terms of "guessing games". Exploring this, we show that relations in a particular algebra-the thermodynamic semiring-for the guessing game operad correspond naturally to information-theoretic properties of the entropy functions, cominiscent of an operadic characterization studied recently by Baez, Fritz and Leinster, which we review. This allows us to rephrase Connes and Consani's original construction in a way that makes clear why the Shannon entropy plays such a key role and provides a categorification of entropy functions.

In the last section we outline possible further directions, some of which will eventually relate back the general theory of thermodynamic semirings to the analogies between characteristic $p$ and characteristic one geometries. Thus, this point of view based on thermodynamic semirings may be regarded as yet another possible viewpoint on $\mathbb{F}_{1}$-geometry, based on information theory and statistical geometry, a sort of "cybernetic viewpoint".

1.1. Witt vectors and their characteristic one analogs. Witt vectors were first proposed by Ernst Witt in 1936 to describe unramified extensions of the $p$-adic numbers. In particular, Witt developed integral polynomial expressions for the arithmetic of strict $p$-rings in terms of their residue rings.

A ring $R$ is a strict $p$-ring when $R$ is complete and Hausdorff under the $p$-adic metric, $p$ is not a zero-divisor in $R$, and the residue ring $K=R / p R$ is perfect [33], [44], [47]. The ring $R$ is determined by $K$ up to canonical isomorphism, and there is a unique multiplicative section $\tau: K \rightarrow R$ of the residue morphism $\pi: R \rightarrow K$, i.e.,

$$
\pi \circ \tau=\operatorname{id}_{K}, \quad \tau(x y)=\tau(x) \tau(y) \quad \text { for all } x, y \in K .
$$


Every element $x$ of $R$ can be written uniquely as

$$
x=\sum \tau\left(x_{n}\right) p^{n}, \quad x_{n} \in K .
$$

The $\tau(x)$ are called Teichmüller representatives.

When $K=\mathbb{F}_{p}, R=\mathbb{Z}_{p}$, but the Teichmüller representatives are not $\{0,1, \ldots, p-$ $1\}$ as they are in the common representation of $\mathbb{Z}_{p}$. Instead they are the roots of $x^{p}-x$. We see from this example that the arithmetic in terms of the Teichmüller representation above is nontrivial. The Witt formula expresses the sum of these representatives as

$$
\tau(x)+\tau(y)=\tilde{\tau}\left(\sum_{\alpha \in I_{p}} w_{p}(\alpha, T) x^{\alpha} y^{1-\alpha}\right),
$$

where $I_{p}=\left\{\alpha \in \mathbb{Q} \cap[0,1] \mid p^{n} \alpha \in \mathbb{Z}\right.$ for some $\left.n\right\}, \tilde{\tau}: K[[T]] \rightarrow R$ is the unique map such that $\tilde{\tau}\left(x T^{n}\right)=\tau(x) p^{n}$, and $w_{p}(\alpha, T) \in \mathbb{F}_{p}[[T]]$ is independent of $R$. Note that, since $K$ is perfect, the terms $x^{\alpha} y^{1-\alpha}$ make sense.

The idea of [10], [11] is to generalize this to characteristic one by considering sums of the form

$$
x \oplus w y:=\sum_{\alpha \in I} w(\alpha) x^{\alpha} y^{1-\alpha}
$$

where now $I=\mathbb{Q} \cap[0,1]$ over sufficiently nice characteristic one semirings.

According to Definition 2.7 of [11], a semiring is characteristic one when $1+1=$ 1 , i.e., when it is idempotent. For example, the tropical semifield, $\mathbb{T}=\mathbb{R} \cup\{-\infty\}$, with addition given by the sup and multiplication given by normal addition, forms a well studied characteristic one semiring in the context of tropical geometry [24], [35].

Connes and Consani found in [10], [11] that, over a suitably nice characteristic one semiring, $\oplus_{w}$ is commutative, associative, shares an identity with + , and is order-preserving if and only if $w(\alpha)$ is of the form

$$
w(\alpha)=\rho^{\mathrm{Sh}(\alpha)},
$$

where $\rho \geqslant 1$ and $\operatorname{Sh}(p)$ is the well-known Shannon entropy

$$
\operatorname{Sh}(p)=-C(p \log p+(1-p) \log (1-p)),
$$

where we write $\log$ for the natural $\log$ arithm, and where $C>0$ is an arbitrary constant factor.

In this paper, we attempt to elucidate this surprising connection between the algebraic structure of the semiring and the information-theoretic entropy by developing a broader theory of thermodynamic semirings.

\section{Preliminary notions}

We introduce here some basic facts that we will need to use in the rest of the paper. 
We start with a warning about notation. Throughout most of the paper we will work implicitly with $\mathbb{R}^{\min ,+} \cup\{\infty\}$ or $\mathbb{R}_{\geqslant 0}^{\max , *}$ in mind (note the two are isomorphic under $-\log$ ). As such, we will use the notation in one of the two. Which one we use should hopefully be clear from the context. We do this because the first will give expressions looking more like statistical physics equations, and the second will give expressions more similar to the Witt construction in characteristic $p$. We will tend to write $\oplus_{S, T}$ (perhaps with other relevant subscripts) for the Witt addition, to indicate that it is a modification of the additive structure of the semiring, and that it depends on the choice of a binary information measure (or entropy) $S$ and of a temperature parameter $T$. This is motivated by tropical geometry, where it is customary to denote by $\oplus$ the addition in the tropical semiring, i.e., the minimum, and by $\odot$ the multiplication, the usual addition + , see [35].

2.1. Frobenius in characteristic one. We recall here, from [10], [11], the behavior of the Frobenius action in the characteristic one setting.

Let $K$ be a commutative, characteristic one semifield. It is possible to work in the slightly more general case of multiplicatively cancellative semirings, but for simplicity we will forsake this generality. Recall that such a semifield is a set with two associative, commutative binary operations, $(x, y) \mapsto x+y$ and $(x, y) \mapsto x y$ such that the second distributes over the first, $0+x=x, 0 x=0,1 x=x, K$ has multiplicative inverses, and, importantly, the characteristic one condition that $1+1=1$.

The first step in developing an analog of the Witt construction is to examine the Frobenius map in $K$.

Lemma 2.1 (Frobenius).

$$
(x+y)^{n}=x^{n}+y^{n} \quad \text { for every } n \in \mathbb{N} .
$$

Proof. The proof is given in Lemma 4.3 of [10], but we recall it here for the convenience of the readers. One sees from the distributive property that, for every $m \in \mathbb{N}$, one has $(x+y)^{m}=\sum_{k=0}^{m} x^{k} y^{m-k}$. This then gives $\left(x^{n}+y^{n}\right)(x+y)^{n-1}=$ $(x+y)^{2 n-1}$. Since $K$ is multiplicatively cancellative, this implies (2.1).

2.2. Legendre transform. As shown in Lemma 4.2 of [10], $K$ is endowed with a natural partial ordering $\leqslant$ defined so that $x \leqslant y \Longleftrightarrow x+y=y$. This may seem strange, but one sees that, over the tropical semifield $\mathbb{T}$, this reads $x \leqslant y \Longleftrightarrow$ $\max (x, y)=y$. We give $K$ the order topology from $\leqslant$. Then multiplication and the Frobenius automorphisms make $K$ a topological $\mathbb{R}_{\geqslant 0}$-module, since the Frobenius is continuous and distributes over the multiplicative structure. When $K=\mathbb{T}$, this topology is the standard one on $[0,1) \cong \mathbb{R} \cup\{-\infty\}$, with the Frobenius acting by multiplication so that $K$ has the normal vector space structure. 
We say that a function $f: X \rightarrow K$, where $X$ is a convex subset of a topological $\mathbb{R} \geqslant 0$-module, is convex if, for every $t \in[0,1], x_{1}, x_{2} \in X$,

$$
f\left(t x_{1}+(1-t) x_{2}\right) \leqslant f\left(x_{1}\right)^{t} f\left(x_{2}\right)^{1-t},
$$

with concavity being defined as convexity of the multiplicative inverse of $f$.

Note again that, over $\mathbb{T}$, this is the normal definition of convexity.

We consider also

$$
\text { epi } f=\{(\alpha, r) \in X \times K \mid f(\alpha) \leqslant r\} \text {, }
$$

called the epigraph of $f$. This has the following property.

Lemma 2.2. A function $f$ is convex iff the epigraph epi $f$ is convex and $f$ is closed iff epi $f$ is closed.

Proof. The topological $\mathbb{R}_{\geqslant 0}$-module structure on $X \times K$ is given by the product structure, so the proof follows directly from the definitions.

When $X \subseteq \mathbb{R}_{\geqslant 0}$, we can define the Legendre transform of $f$ by

$$
f^{*}(x)=\sum_{\alpha \in X} \frac{x^{\alpha}}{f(\alpha)} .
$$

Note that over $\mathbb{T}$ this reads

$$
\sup _{\alpha \in X}(\alpha x-f(\alpha)),
$$

which is the normal definition of the Legendre transform.

When $X \subseteq K$, we can define the Legendre transform of $f$ by

$$
f^{*}(\alpha)=\sum_{x \in X} \frac{x^{\alpha}}{f(x)} .
$$

Proposition 2.3. The Legendre transform of $f$ is closed and convex.

Proof. Suppose first that $X \subset \mathbb{R}_{\geqslant 0}$. Let $g_{\alpha}(x)=x^{\alpha} / f(\alpha)$, and $g$ be the Legendre transform of $f$. Then $g$ is the point-wise supremum among the $g_{\alpha}$, so epi $g=$ $\bigcap_{\alpha \in X}$ epi $g_{\alpha}$, an intersection of closed half spaces. Thus, epi $g$ is closed and convex, so $g$ is closed and convex, by the Lemma 2.2. The proof of the opposite case proceeds in precisely the same manner.

One then has the following result on Legendre transforms.

Theorem 2.4 (Fenchel-Moreau). Let $f: X \rightarrow K, X \subset \mathbb{R}_{\geqslant 0}$. Then the following hold. 
(1) $f^{* *}$ is closed and convex and bounded by $f$.

(2) $f^{* *}=f$ iff $f$ is closed and convex.

Proof. The function $f^{* *}$ is convex and closed by Lemma 2.2. We also see that

$$
x^{\alpha} / f^{*}(x) \leqslant x^{\alpha} /\left(x^{\alpha} / f(\alpha)\right)=f(\alpha),
$$

so taking a $\sup _{x \in X}$ of both sides yields $f^{* *} \leqslant f$. To prove the second fact, it suffices to show that, if $f$ is closed, convex, and finite, then $f \leqslant f^{* *}$. Define the subdifferential $\partial f(\alpha)$ of $f$ at $\alpha$ by

$$
\partial f(\alpha)=\left\{x \in R \mid f(\beta) \geqslant f(\alpha) x^{\beta-\alpha} \text { for all } \beta \in X\right\} .
$$

We consider the set-valued map $\alpha \mapsto \partial f(\alpha)$. To invert this map is to find $\alpha(x)=\alpha$ such that $x \in \partial f(\alpha)$. We see that $f^{*}(x)=x^{\alpha(x)} / f(\alpha(x))$. Thus, the subdifferential is the proper analog in this case for the derivative. When $f$ is closed and convex, $\partial f(\alpha)$ is nonempty, so let $x \in \partial f(\alpha)$. Then we have

$$
\left.1 / f^{*}(x) \geqslant f(\alpha) / x^{\alpha} \Longrightarrow f(\alpha) \leqslant x^{\alpha} / f^{*}(x)\right) \leqslant f^{* *}(\alpha)
$$

for every $\alpha$, proving the theorem.

This is a simple translation of the well-known Legendre transform machinery into characteristic one semirings. The idea is that since we can define a real topological vector space structure on $K$ using the multiplication as addition and the Frobenius map as scalar multiplication (with negative reals having a well-defined action since $K$ has multiplicative inverses), we have enough structure to do convex analysis. The point is that for concave or convex $f$, the above sums are invertible in $K$. From now on, any semifield satisfying the assumptions necessary for this section will be called "suitably nice".

2.3. Witt ring construction in characteristic one. We recall here the main properties of the characteristic one analog of the Witt construction [10], [11], which is the starting point for our work. We formulate it here in terms of a general information measure $S$, whose properties we will find are related to the algebraic properties of the semiring.

Let $w:[0,1] \rightarrow K$ be continuous under the order topology, and consider, for each $x, y \in K$,

$$
x \oplus_{w} y=\sum_{\alpha \in I} w(\alpha) x^{\alpha} y^{1-\alpha} .
$$

Connes and Consani considered the above expression for continuous $w(\alpha) \geq 1$ and found in [10], [11] that $\oplus_{w}$ is commutative, associative and has identity 0 if and only if $w(\alpha)=\rho^{\operatorname{Sh}(\alpha)}$ for some $\rho \in K$ greater than one. 
For simplicity and clarity of intention, we will write $\rho=e^{T}$ for some $T \geqslant 0$ to suggest $T$ behaves like a temperature parameter. In all the arguments that follow, one could replace $e^{T}$ by $\rho$ again and be fine over the more general semifields.

Correspondingly, we are going to restrict our attention to sums of the form

$$
x \oplus S y:=\sum_{\alpha \in I} e^{T S(\alpha)} x^{\alpha} y^{1-\alpha}
$$

where $S$ will be interpreted as an entropy function. In particular, we assume $S$ is concave and closed, so that $e^{-T S(\alpha)}$ is convex and closed, and we can use the Legendre transform machinery developed in $\$ 2.2$.

We can then formulate the result of [11] on the characteristic one Witt construction in the following way.

Theorem 2.5. Suppose that $S: I \rightarrow \mathbb{R}_{\geqslant 0}$ is concave and closed. The following hold.

(1) $x \oplus_{S} y=y \oplus_{S} x$ for all $x, y \in K$ iff $S(\alpha)=S(1-\alpha)$.

(2) $0 \oplus_{S} x=x$ for all $x \in K$ iff $S(0)=0$.

(3) $x \oplus_{S} 0=x$ for all $x \in K$ iff $S(1)=0$.

(4) $x \oplus_{S}\left(y \oplus_{S} z\right)=\left(x \oplus_{S} y\right) \oplus_{S} z$ for all $x, y, z \in K$ iff $S(\alpha \beta)+(1-$ $\alpha \beta) S\left(\frac{\alpha(1-\beta)}{1-\alpha \beta}\right)=S(\alpha)+\alpha S(\beta)$.

Proof. The argument is given in [10] in a more general form applicable to a binary operation as in (1.1), but we give the explicit proof here to show the machinery.

(1) Since $S$ is concave and closed, $e^{-T S}$ is convex and closed (in the generalized sense of (2.2)), as is $z^{L(\alpha)}$ for any linear function $L(\alpha)$ and $z \in K$. We also see that products of convex and closed functions are convex and closed, so $y^{\alpha-1} e^{-T S(\alpha)}$ and $y^{\alpha-1} e^{-T S(1-\alpha)}$ are each convex and closed. We see that $x \oplus_{S} y=y \oplus_{S} x$ iff

$$
\sum_{\alpha \in I} \frac{x^{\alpha}}{y^{\alpha-1} e^{-T S(\alpha)}}=\sum_{\alpha \in I} \frac{x^{\alpha}}{y^{\alpha-1} e^{-T S(1-\alpha)}} .
$$

We recognize the Legendre transform of closed convex functions, which is invertible by the Fenchel-Moreau theorem above. Thus, the summands must be equal, so $S(\alpha)=S(1-\alpha)$. The converse is obvious.

(2) First note that, when $\alpha \neq 0$, for every $x, 0^{\alpha} x^{1-\alpha}=0$ and $e^{T S(0)} \geq 0$, so the supremum occurs at $\alpha=0$. Therefore, we have $0 \oplus S x=e^{T S(0)} x$.

(3) Similarly, this supremum occurs at $\alpha=1$, so $x \oplus_{S} 0=e^{T S(1)} x$.

(4) As in fact 1 , we see that $x \oplus_{S}\left(y \oplus_{S} z\right)=\left(x \oplus_{S} y\right) \oplus_{S} z$ iff

$$
\sum_{\alpha, \beta \in I} \frac{x^{\alpha \beta}}{y^{\alpha(\beta-1)} z^{\alpha-1} e^{-T(S(\alpha)+\alpha S(\beta))}}=\sum_{u, v \in I} \frac{x^{u}}{y^{v(u-1)} z^{(v-1)(1-u)} e^{-T(S(u)+(1-u) S(v))}} .
$$

Identifying powers and inverting the Legendre transform yields the condition. The converse is immediate. 
We hold off discussing the fact that the Shannon entropy Sh is the only function $S$ satisfying all of these properties until $\S 3$ below, where we develop the informationtheoretic interpretation of these axioms.

\section{Axioms for entropy functions}

It is well known that the Shannon entropy admits an axiomatic characterization in terms of the Khinchin axioms [26]. These are usually stated as follows for an information measure $S\left(p_{1}, \ldots, p_{n}\right)$ :

(1) (Continuity) For any $n \in \mathbb{N}$, the function $S\left(p_{1}, \ldots, p_{n}\right)$ is continuous with respect to $\left(p_{1}, \ldots, p_{n}\right)$ in the simplex $\Delta_{n}=\left\{p_{i} \in \mathbb{R}_{+}, \sum_{i} p_{i}=1\right\}$;

(2) (Maximality) Given $n \in \mathbb{N}$ and $\left(p_{1}, \ldots, p_{n}\right) \in \Delta_{n}$, the function $S\left(p_{1}, \ldots, p_{n}\right)$ has its maximum at the uniform distribution $p_{i}=1 / n$ for all $i=1, \ldots, n$,

$$
S\left(p_{1}, \ldots, p_{n}\right) \leq S\left(\frac{1}{n}, \ldots, \frac{1}{n}\right) \quad \text { for all }\left(p_{1}, \ldots, p_{n}\right) \in \Delta_{n} ;
$$

(3) (Additivity) If $p_{i}=\sum_{j=1}^{m_{i}} p_{i j}$ with $p_{i j} \geq 0$, then

$$
S\left(p_{11}, \ldots, p_{n m_{n}}\right)=S\left(p_{1}, \ldots, p_{n}\right)+\sum_{i=1}^{n} p_{i} S\left(\frac{p_{i 1}}{p_{i}}, \ldots, \frac{p_{i m_{i}}}{p_{i}}\right) ;
$$

(4) (Expandability) Embedding a simplex $\Delta_{n}$ as a face inside a simplex $\Delta_{n+1}$ has no effect on the entropy,

$$
S\left(p_{1}, \ldots, p_{n}, 0\right)=S\left(p_{1}, \ldots, p_{n}\right) .
$$

It is shown in [26] that there is a unique information measure $S\left(p_{1}, \ldots, p_{n}\right)$ (up to a multiplicative constant $C>0$ ) that satisfies these axioms and it is given by the Shannon entropy

$$
S\left(p_{1}, \ldots, p_{n}\right)=\operatorname{Sh}\left(p_{1}, \ldots, p_{n}\right):=-C \sum_{i=1}^{n} p_{i} \log p_{i},
$$

We focus now on the $n=2$ case, which means that we are only looking at $S(p):=S(p, 1-p)$ instead of the more general $S\left(p_{1}, \ldots, p_{n}\right)$. In other words, we are only considering the information theory of binary random variables. In this case, we describe here an axiomatic formulation for the Shannon entropy based on properties of binary "decision machines". We return to discuss the more general $n$-ary case in $\S 10$ below. 
A decision machine is a measurement tool which may only distinguish between two possible states of a discrete random variable; machines that can only answer "yes" or "no". We would like to measure the average change in uncertainty after a measurement, which is how we define the entropy associated with a random variable. Let $X$ be a binary random variable, $S(X)$ the change in entropy after measuring $X$. All information is created equal, so $S(X)$ should only depend on the probability of measuring a certain value of $X$ and should do so continuously.

(1) (Left identity) $S(0)=0$.

(2) (Right identity) $S(1)=0$.

(3) (Commutativity) $S(p)=S(1-p)$.

(4) (Associativity) $S\left(p_{1}\right)+\left(1-p_{1}\right) S\left(\frac{p_{2}}{1-p_{1}}\right)=S\left(p_{1}+p_{2}\right)+\left(p_{1}+p_{2}\right) S\left(\frac{p_{1}}{p_{1}+p_{2}}\right)$.

The identity axioms claim that trivial measurements give trivial information.

The commutativity axiom claims that questions have the same information as their negative.

The associativity axiom claims a certain equivalence of guessing strategies, which will be a key observation in our explanation of the characteristic one Witt construction. If instead of a binary random variable, we want to measure a ternary random variable $X$ which may take values $X \in\left\{x_{1}, x_{2}, x_{3}\right\}$ with corresponding probabilities $p_{1}, p_{2}, p_{3}$, we can still determine $X$ by asking yes-or-no questions. We can first ask "is $X=x_{1}$ ?" If the answer is no (which occurs with probability $p_{2}+p_{3}$ ), we then ask "is $X=x_{2}$ ?" This corresponds to an average change in uncertainty $S\left(p_{1}\right)+\left(p_{2}+p_{3}\right) S\left(\frac{p_{2}}{p_{2}+p_{3}}\right)$. However, we could have asked "is $X=x_{1}$ or $x_{2}$ ?" followed by "is $X=x_{1}$ ?" and in the end received the same data about $X$. Associativity asserts these two should be equal, hence we have the axiom as stated above.

The names of the axioms in the above list are chosen to suggest the corresponding algebraic properties, as we see in Theorem 4.2 below. In fact, we find that these algebraically motivated axioms are equivalent to the Khinchin axioms.

Theorem 3.1. There is a unique function (up to a multiplicative constant $C>0$ ) satisfying all of the axioms above, namely the Shannon entropy

$$
\operatorname{Sh}(p)=-C(p \log p+(1-p) \log (1-p)) .
$$

Proof. The result follows either by checking directly the equivalence of the commutativity, identity and associativity axioms with the Khinchin axioms, or else by proceeding as in Theorem 5.3 of [10]. We prove it here by showing that one obtains the Khinchin axioms for entropy.

Suppose that $S$ satisfies all the conditions above. Define $S_{n}: \Delta_{n-1} \rightarrow \mathbb{R}_{\geqslant 0}$ by

$$
S_{n}\left(p_{1}, \ldots, p_{n}\right)=\sum_{1 \leqslant j \leqslant n-1}\left(1-\sum_{1 \leqslant i<j} p_{i}\right) S\left(\frac{p_{j}}{1-\sum_{1 \leqslant i<j} p_{i}}\right) .
$$


Lemma 3.2. $S_{n}$ is symmetric.

Proof. Suppose we interchange the terms $p_{k}$ and $p_{k+1}$, where $k<n-1$. This only affects the $k$-th terms and $(k+1)$-th terms, so we must show that

$$
T=\left(1-\sum_{i<k} p_{i}\right) S\left(\frac{p_{k}}{1-\sum_{i<k} p_{i}}\right)+\left(1-\sum_{i<k+1} p_{i}\right) S\left(\frac{p_{k+1}}{1-\sum_{i<k+1} p_{i}}\right)
$$

is symmetric. Write $\beta=1-\sum_{i<k} p_{i}, a=p_{k} / \beta, b=p_{k+1} / \beta$. We see that $\beta$ is invariant under this permutation, and

$$
T=\beta(S(a)+(1-a) S(b /(1-a))) .
$$

Permuting $p_{k}$ and $p_{k+1}$ interchanges $a$ and $b$, and so $T$ is invariant by the associativity condition. Interchanging $p_{n-1}$ and $p_{n}$ only affects the last term, and it is easy to see it affects it like $S(\alpha) \mapsto S(1-\alpha)$, so invariance follows from commutativity. These transpositions generate the symmetric group $\operatorname{Sym}_{n}$, so $S_{n}$ is symmetric.

From this lemma and the definition we see that the following holds.

Lemma 3.3. Let $\left(J_{k}\right)_{1 \leqslant k \leqslant m}$ be a partition of $\left\{p_{1}, \ldots, p_{n}\right\}$ and let $S_{n}$ be defined as in (3.1). Then we have

$$
S_{n}\left(p_{1}, \ldots, p_{n}\right)=S_{m}\left(q_{1}, \ldots, q_{m}\right)+\sum_{1 \leqslant k \leqslant m} S_{\left|J_{k}\right|}\left(J_{k} / q_{k}\right),
$$

where $q_{k}=\sum_{p \in J_{k}} p$, so $J_{k} / q_{k}$ is a $\left|J_{k}\right|$-ary probability distribution.

These lemmas take care of the third Khinchin axiom, and with the identity property also take care of the fourth. We assumed at the outset $S$ was continuous, so it follows from the definition $S_{n}$ is continuous, which is the first axiom. What remains is the second axiom, which we write here in terms of information (concave) rather than entropy (convex).

Lemma 3.4. $S_{n}$ is concave for all $n$.

Proof. We proceed by induction on $n$. We have already assumed $S_{2}=S$ is concave, so suppose that $S_{n}$ is concave for some $n \geq 2$. Note that for continuous $f$, concavity follows from $f\left(\frac{x+y}{2}\right) \geq \frac{f(x)+f(y)}{2}$. Thus we consider, for some $\left(p_{i}\right),\left(q_{i}\right) \in \Delta_{n+1}$,

$$
S_{n+1}\left(\frac{p_{1}}{2}+\frac{q_{1}}{2}, \ldots, \frac{p_{n+1}}{2}+\frac{q_{n+1}}{2}\right) .
$$

By the previous lemma, this equals

$$
\begin{gathered}
S_{n}\left(\frac{p_{1}+p_{2}}{2}+\frac{q_{1}+q_{2}}{2}, \frac{p_{3}}{2}+\frac{q_{3}}{2}, \ldots, \frac{p_{n+1}}{2}+\frac{q_{n+1}}{2}\right) \\
+\frac{p_{1}+p_{2}+q_{1}+q_{2}}{2} S\left(\frac{p_{1}+q_{1}}{p_{1}+p_{2}+q_{1}+q_{2}}\right) .
\end{gathered}
$$


By the inductive hypothesis we then have

$$
\begin{aligned}
S_{n+1}(\ldots) \geq & \frac{1}{2} S\left(p_{1}+p_{2}, \ldots, p_{n+1}\right)+\frac{1}{2} S\left(q_{1}+q_{2}, \ldots, q_{n+1}\right) \\
& +\frac{p_{1}+p_{2}+q_{1}+q_{2}}{2} S\left(\frac{p_{1}+q_{1}}{p_{1}+p_{2}+q_{1}+q_{2}}\right) .
\end{aligned}
$$

We see that

$\frac{p_{1}+q_{1}}{p_{1}+p_{2}+q_{1}+q_{2}}=\frac{p_{1}+p_{2}}{p_{1}+p_{2}+q_{1}+q_{2}} \frac{p_{1}}{p_{1}+p_{2}}+\frac{q_{1}+q_{2}}{p_{1}+p_{2}+q_{1}+q_{2}} \frac{q_{1}}{q_{1}+q_{2}}$ and

$$
\frac{p_{1}+p_{2}}{p_{1}+p_{2}+q_{1}+q_{2}}+\frac{q_{1}+q_{2}}{p_{1}+p_{2}+q_{1}+q_{2}}=1,
$$

so by the concavity of $S$ we have

$$
\begin{aligned}
S_{n+1}(\ldots) \geq & \frac{1}{2} S\left(p_{1}+p_{2}, \ldots, p_{n+1}\right)+\frac{1}{2} S\left(q_{1}+q_{2}, \ldots, q_{n+1}\right) \\
& +\frac{p_{1}+p_{2}}{2} S\left(\frac{p_{1}}{p_{1}+p_{2}}\right)+\frac{q_{1}+q_{2}}{2} S\left(\frac{q_{1}}{q_{1}+q_{2}}\right),
\end{aligned}
$$

from which concavity of $S_{n+1}$ follows by the previous lemma.

Since $S_{n}$ is concave, it has a unique maximum, and since it is symmetric, this maximum occurs at $S_{n}\left(\frac{1}{n}, \ldots, \frac{1}{n}\right)$, implying the second Khinchin axiom. This then completes the proof of Theorem 3.1

A reformulation of the Khinchin axioms for Shannon entropy more similar to the commutativity, identity and associativity axioms considered here was described in Faddeev's [17]. For different reformulations of the Khinchin axioms see also [13].

\section{Thermodynamic semirings}

We now consider more general thermodynamic semirings. The following definition describes the basic structure.

Definition 4.1. A thermodynamic semiring structure over $K$, written $\mathbb{R}^{\min ,+} \cup\{\infty\}$, is a collection of binary operations $\oplus S, T: K \times K \rightarrow K$ indexed by $T \in \mathbb{R} \cup\{\infty\}$ and defined by an information measure $S:[0,1] \rightarrow \mathbb{R}$ according to

$$
x \oplus S, T y=\min _{p \in[0,1] \cap \mathbb{Q}}(p x+(1-p) y-T S(p)) .
$$


It is often convenient to consider the elements of the semiring as functions of $T$, with the operation $\oplus_{S}$ defined pointwise by $\oplus_{S, T}$. We call this ring $R$, inspired the $p$-typical Witt notation. Indeed in [11], [10], $R$ is seen as the Witt ring over $K$, with evaluation at $T=0$ over giving the residue morphism $R \rightarrow K$. We then see that the Teichmüller lifts should be the constant functions, and $T$ should play the role of the exponent of $p^{n}$ in considering field extensions.

We then have the following general properties, as in Theorem 3.1 above (Theorem 5.2 of [10]):

Theorem 4.2. Let $x \oplus_{S, T} y$ be a thermodynamic semiring structure on a suitably nice characteristic one semifield, $K$, defined as in (4.1). Then the following holds.

(1) $x \oplus_{S, T} y=y \oplus_{S, T} x$ iff $S$ is commutative.

(2) $0 \oplus S, T x=x$ iff $S$ has the left identity property.

(3) $x \oplus_{S, T} 0=x$ iff $S$ has the right identity property.

(4) $x \oplus_{S, T}\left(y \oplus_{S, T} z\right)=\left(x \oplus_{S, T} y\right) \oplus_{S, T} z$ iff $S$ is associative.

Proof. The case of commutativity and of the identity axioms are obvious. For associativity we have

$$
\begin{aligned}
x & \oplus S, T \\
& =x \oplus_{S, T} \min _{p}(p y+(1-p) z-T S(p)) \\
& =\min _{q}\left(q x+(1-q) \min _{p}(p y+(1-p) z-T S(p))-T S(q)\right) \\
& =\min _{p, q}(q x+p(1-q) y+(1-q)(1-p) z-T(S(q)+(1-q) S(p))) \\
& =\min _{p_{1}+p_{2}+p_{3}=1}\left(p_{1} x+p_{2} y+p_{3} z-T\left(S\left(p_{1}\right)+\left(1-p_{1}\right) S\left(\frac{p_{2}}{1-p_{1}}\right)\right)\right),
\end{aligned}
$$

while

$$
\begin{aligned}
(x & \oplus S, T y) \oplus S, T z \\
& =\min _{p}(p x+(1-p) y-T S(p)) \oplus_{S} z \\
& =\min _{p, q}(p q x+q(1-p) y+(1-q) z-T(q S(p)+S(q)) \\
& =\min _{p_{1}+p_{2}+p_{3}=1}\left(p_{1} x+p_{2} y+p_{3} z-T\left(S\left(p_{1}+p_{2}\right)+\left(p_{1}+p_{2}\right) S\left(\frac{p_{1}}{p_{1}+p_{2}}\right)\right) .\right.
\end{aligned}
$$

We see that the two ways of summing three quantities corresponds to the two ways of measuring a ternary random variable with decision machines. The equivalence is now obvious.

Most information measures are commutative, though a non-commutative example in $\S 8$ below. We discuss in $\S 5$ some physical reasons why commutativity is more automatic in this context than associativity. 
One then sees by direct inspection that, in the case of the Shannon entropy one has the following form of the thermodynamic semiring structure.

Proposition 4.3. When $S$ is the Shannon entropy, Sh, then

$$
x \oplus_{\mathrm{Sh}, T} y=-T \log \left(e^{-x / T}+e^{-y / T}\right)
$$

over $\mathbb{R}^{\min ,+} \cup\{\infty\}$, while over $\mathbb{R}_{\geqslant 0}^{\max , *}$ it is

$$
x \oplus_{\mathrm{Sh}, T} y=\left(x^{1 / T}+y^{1 / T}\right)^{T} .
$$

Notice that the semiring $\mathbb{R}_{\geqslant 0}^{\max , *}$ is isomorphic to the semiring $\mathbb{R}^{\min ,+} \cup\{\infty\}$, under the - log mapping, so that (4.3) is simply obtained from (4.2) in this way.

In this case, the parameter $T$ corresponds to the parameter $h$ of Maslov dequantization (see the comments in $§ 11.2$ ). The semifields obtained in this way are known as the Gibbs-Maslov semirings and the subtropical algebra (see [34], [31]).

One can extend the notion of thermodynamic semiring to include a class of semirings of functions which we will be considering in the following. Just as in the case of a ring $R$ and a parameter space $X$, one can endow the set of functions from $\Xi$ to $R$ with a ring structure, by pointwise operations, one can proceed similarly with a semiring. Moreover, in the case of a thermodynamic semiring structure, it is especially interesting to consider cases where the pointwise operation $\oplus_{S, T}$ depends on the point in the parameter space through a varying entropy function $S=S_{\eta}$ for $\eta \in \Xi$.

Definition 4.4. Let $\Xi$ be a compact Hausdorff space and let $S=\left(S_{\eta}\right)$ be a family of information measures depending continuously on the parameter $\eta \in \Xi$. Let $K=\mathbb{R}^{\mathrm{min},+} \cup\{\infty\}$. A thermodynamic semiring structure on the space of functions $C(X, R)$ is given by the family of pointwise operations

$$
x(\eta) \oplus S_{\eta}, T y(\eta)=\min _{p \in[0,1] \cap \mathbb{Q}}\left(p x(\eta)+(1-p) y(\eta)-T S_{\eta}(p)\right) .
$$

The properties of Theorem 4.2 extend to this case. We will return to this more general setting in $\$ 8$ below.

As we discuss in the following sections, more general entropy functions (which include the special cases of Rényi entropy, Tsallis entropy and Kullback-Leibler divergence, as well as the more general categorical and operadic setting developed in $§ 10)$ give rise to thermodynamic algebraic structures that are neither commutative nor associative. We will continue to use the terminology "semiring", although (as the referee pointed out to us) the term "algebra", in the sense of the theory of universal algebra, would be more appropriate. 


\section{Statistical mechanics}

Before we move on to see explicit examples of thermodynamic semirings besides the original one based on the Shannon entropy considered already in [10] and [11], we give in this section a physical interpretation of the algebraic structure of thermodynamic semirings in terms of statistical mechanics. This interpretation is a generalization of thermodynamic interpretations of max-plus formulas found in [43]. form

When $K=\mathbb{R}_{\geqslant 0}^{\max , *}$, we can write the thermodynamic semiring operations in the

$$
x \oplus_{\rho, S} y=\max _{p}\left(\rho^{S(p)} x^{p} y^{1-p}\right) .
$$

In particular, when we set $\rho=e^{k_{B} T}$, this reads

$$
\max _{p}\left(e^{k_{B} T S(p)+p \log x+(1-p) \log y}\right) .
$$

We recognize this as $e^{-F_{\mathrm{eq}}}=Z$, where $F_{\mathrm{eq}}$ is the equilibrium value of the free energy of a system at temperature $T$, containing a gas of particles with chemical potentials $\log x$ and $\log y$, and Hamiltonian

$$
\mathscr{H}=p \log x+(1-p) \log y,
$$

where $p$ is now thought of as a mole fraction, and $Z$ is its partition function.

Indeed, the semirings $\mathbb{R}_{\geqslant 0}^{\max , *}$ and $\mathbb{R}^{\min ,+} \cup\{\infty\}$ are isomorphic by $-\log$, and this gives

$$
\log x \oplus_{S, k_{B} T} \log y=\min _{p}\left(p \log x+(1-p) \log y-k_{B} T S(p)\right),
$$

which is the equilibrium free energy described above. We note also that the calculated form of the thermodynamic semiring for Shannon entropy, that is

$$
x \oplus \operatorname{Sh} y=-T \log \left(e^{-x / T}+e^{-y / T}\right) .
$$

In it, we recognize precisely the partition sum of a two state system with energies $x$ and $y$. We thus consider members of the Witt ring $R$ (see §4) to be temperature dependent chemical potentials.

In a gas system with a single type of particle, the free energy is precisely the chemical potential. The mixing of these gases gives a new free energy dependent on the entropy function. We then replace this mixture with a "particle" whose chemical potential is the equilibrium free energy per particle of the previous mixture. This gives a monoid structure on the space of chemical potentials. When we consider mixing in arbitrary thermodynamics, i.e., with non-Boltzmann counting, we have the possibility of mixing to be non-associative. With this interpretation, however, we would not expect the mixing process to ever be non-commutative, so the lack of associativity has a more direct and natural physical interpretation than the lack of 
commutativity for thermodynamic semirings. We imagine multiplication to be a sort of bonding of gases, where chemical potentials add together.

We see that the dynamics of this mixing process is determined, both physically and algebraically, by the entropy function and the ambient temperature. At zero temperature, the mixture is always entirely composed of the particle with the least chemical potential. This corresponds to $\mathbb{R}^{\mathrm{min},+}$ and indeed evaluation at zero temperature gives us the residue morphism $R \rightarrow K$. When the entropy function is the Shannon entropy, we get the normal thermodynamical mixing, see $\$ 8.5$ of [16]. We can say, therefore, that the Witt construction is, in a sense, giving thermodynamics to this system. Note that in (1.1) this construction is seen giving an inverse to Maslov dequantization, pointing out an interesting link between quantum mechanics and thermodynamics.

The mixing entropy for chemical systems based on the Boltzmann-Gibbs statistical mechanics and the Shannon entropy function (as in $\$ 8.5$ of [16] for instance) works well to describe systems that are ergodic. If a system is nonergodic (that is, time averages and phase space averages differ), then the counting involved in bringing two initially separated systems into contact will not follow the normal Boltzmann rules. As a result, Shannon entropy will not behave extensively in these systems. This typically occurs in physical systems with strong, long-range coupling and in systems with metastable states or exhibiting power law behavior. In such systems, maximizing the Shannon entropy functional (subject to the dynamical constraints of the system) does not produce the correct metaequilibrium distribution, see for instance [53] and other essays in the collection [21].

A broad field of non-extensive statistical mechanics for such systems has been developed (see [53] for a brief introduction), where, under suitable conditions, one can calculate a "correct" entropy functional corresponding to the system at hand. These entropy functionals are typically characterized by some axiomatic properties that describe their behavior. For instance, if we have two initially independent systems $A, B$ and bring them together to form a combined system denoted by $A \star B$, one may require that $S(A \star B)=S(A)+S(B)$ (extensive). This leads to forms of entropy such as the Rényi entropy [45], generalizing the original Shannon case, while maintaining the extensivity over independent systems. One may also have explicit $q$-deformations of the extensivity condition, for example $S_{q}(A \star B)=S_{q}(A)+S_{q}(B)+(1-$ q) $S_{q}(A) S_{q}(B)$ for independent systems. This leads to forms of entropy such as the Tsallis entropy [52].

When we consider different kinds of entropy functions in this way, we can look at the algebraic properties of the corresponding thermodynamic semirings. These will encode the information about the amount of nonextensivity and nonergodicity of the system giving rise to the corresponding entropy function $S$. We can imagine nonassociativity of mixing as a toy model of meta-equilibrium states where we known the entropy beforehand. We can also use thermodynamic semirings to encode relative entropies and analyze its behavior over a space of parameters through the algebraic properties of the semiring. 
Relations between idempotent semifields and statistical mechanics were also considered in [23], [25], [43].

\section{The Rényi entropy}

We now look at other important examples of entropy functions and we investigate how the corresponding algebraic properties of the associated thermodynamic semiring detect the properties of the entropy function as an information measure.

A first well-known case of an entropy function which is a natural generalization of the Shannon entropy: the Rényi entropy, [45]. This is a one-parameter family $\mathrm{Ry}_{\alpha}$ of information measures defined by

$$
\operatorname{Ry}_{\alpha}\left(p_{1}, \ldots, p_{n}\right):=\frac{1}{1-\alpha} \log \left(\sum_{i} p_{i}^{\alpha}\right),
$$

so that the limit

$$
\lim _{\alpha \rightarrow 1} \operatorname{Ry}_{\alpha}\left(p_{1}, \ldots, p_{n}\right)=\operatorname{Sh}\left(p_{1}, \ldots, p_{n}\right)
$$

recovers the Shannon entropy. The Rényi entropy has a broad range of applications, especially in the analysis of multifractal systems [6], while a statistical mechanics based on the Rényi entropy is described in [29].

The Rényi entropy also has an axiomatic characterization, where one weakens the Khinchin additivity axioms to a form that only requires additivity of the information entropy for independent subsystems, while keeping the other three axioms unchanged, [46]. For our version of the axioms, formulated in terms of decision machines, this means that the associativity axiom no longer holds.

Lemma 6.1. The lack of associativity of $x \oplus_{S} y$, when $S=\mathrm{Ry}_{\alpha}$ is the Renyi entropy

$$
\operatorname{Ry}_{\alpha}(p)=\frac{1}{1-\alpha} \log \left(p^{\alpha}+(1-p)^{\alpha}\right),
$$

is measured by the transformation $\left(p_{1}, p_{2}, p_{3}\right) \mapsto\left(p_{3}, p_{2}, p_{1}\right)$.

Proof. We have

$$
\begin{aligned}
& \operatorname{Ry}_{\alpha}\left(p_{1}\right)+\left(1-p_{1}\right) \operatorname{Ry}_{\alpha}\left(\frac{p_{2}}{1-p_{1}}\right) \\
& =\frac{1}{1-\alpha}\left(\log \left(p_{1}^{\alpha}+\left(1-p_{1}\right)^{\alpha}\right)+\left(1-p_{1}\right) \log \left(\left(\frac{p_{2}}{1-p_{1}}\right)^{\alpha}+\left(\frac{1-p_{1}-p_{2}}{1-p_{1}}\right)^{\alpha}\right)\right) \\
& =\frac{1}{1-\alpha} \log \left(\left(p_{1}^{\alpha}+\left(1-p_{1}\right)^{\alpha}\right) \frac{\left(\frac{p_{2}}{1-p_{1}}\right)^{\alpha}+\left(\frac{p_{3}}{1-p_{1}}\right)^{\alpha}}{\left(\left(\frac{p_{2}}{1-p_{1}}\right)^{\alpha}+\left(\frac{p_{3}}{1-p_{1}}\right)^{\alpha}\right)^{p_{1}}}\right) \\
& =\frac{1}{1-\alpha} \log \left(\left(\frac{p_{1} p_{2}}{1-p_{1}}\right)^{\alpha}+\left(\frac{p_{1} p_{3}}{1-p_{1}}\right)^{\alpha}+p_{2}^{\alpha}+p_{3}^{\alpha}\right)
\end{aligned}
$$




$$
\begin{aligned}
& -\frac{p_{1}}{1-\alpha} \log \left(\left(\frac{p_{2}}{1-p_{1}}\right)^{\alpha}+\left(\frac{p_{3}}{1-p_{1}}\right)^{\alpha}\right) \\
& \frac{1}{1-\alpha} \log \left(\frac{\left(p_{2}^{\alpha}+p_{3}^{\alpha}\right)\left(p_{1}^{\alpha}+\left(1-p_{1}\right)^{\alpha}\right)}{\left(1-p_{1}\right)^{\alpha}}\right)-\frac{p_{1}}{1-\alpha} \log \left(\frac{\left(p_{2}^{\alpha}+p_{3}^{\alpha}\right)}{\left(1-p_{1}\right)^{\alpha}}\right) \\
= & \frac{1}{1-\alpha}\left(\left(1-p_{1}\right) \log \left(p_{2}^{\alpha}+p_{3}^{\alpha}\right)\right. \\
& \left.+\log \left(p_{1}^{\alpha}+\left(1-p_{1}\right)^{\alpha}\right)-\alpha\left(1-p_{1}\right) \log \left(1-p_{1}\right)\right) .
\end{aligned}
$$

On the other hand, we have

$$
\begin{aligned}
& \operatorname{Ry}_{\alpha}\left(p_{1}+p_{2}\right)+\left(p_{1}+p_{2}\right) \operatorname{Ry}_{\alpha}\left(\frac{p_{1}}{p_{1}+p_{2}}\right) \\
&=\operatorname{Ry}_{\alpha}\left(1-p_{3}\right)+\left(1-p_{3}\right) \operatorname{Ry}_{\alpha}\left(\frac{p_{1}}{1-p_{3}}\right) \\
&= \operatorname{Ry}_{\alpha}\left(p_{3}\right)+\left(1-p_{3}\right) \operatorname{Ry}_{\alpha}\left(\frac{p_{1}}{1-p_{3}}\right) \\
&= \frac{1}{1-\alpha} \log \left(\left(\frac{p_{1} p_{3}}{1-p_{3}}\right)^{\alpha}+\left(\frac{p_{2} p_{3}}{1-p_{3}}\right)^{\alpha}+p_{1}^{\alpha}+p_{2}^{\alpha}\right) \\
&-\frac{p_{3}}{1-\alpha} \log \left(\left(\frac{p_{1}}{1-p_{3}}\right)^{\alpha}+\left(\frac{p_{2}}{1-p_{3}}\right)^{\alpha}\right) \\
&= \frac{1}{1-\alpha}\left(\left(1-p_{3}\right) \log \left(p_{2}^{\alpha}+p_{1}^{\alpha}\right)+\log \left(p_{3}^{\alpha}+\left(1-p_{3}\right)^{\alpha}\right)\right. \\
&\left.-\alpha\left(1-p_{3}\right) \log \left(1-p_{3}\right)\right) .
\end{aligned}
$$

So the failure of associativity is corrected by mapping $\left(p_{1}, p_{2}, p_{3}\right) \mapsto\left(p_{3}, p_{2}, p_{1}\right)$. In fact, this holds for any commutative $S$.

In a commutative non-associative semiring $K$, the lack of associativity is corrected by the morphism

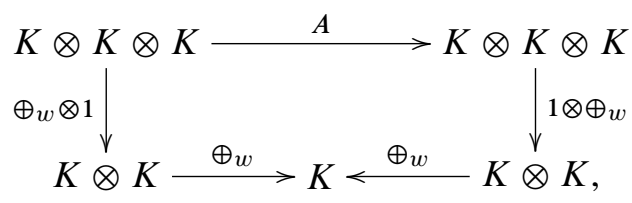

which makes the diagram commutative and which is simply given by $A(x \otimes y \otimes z)=$ $z \otimes y \otimes x$. This is exactly the transformation $\left(p_{1}, p_{2}, p_{3}\right) \mapsto\left(p_{3}, p_{2}, p_{1}\right)$, as these correspond to $p_{1}=s r, p_{2}=s(1-r)$ and $p_{3}=1-\left(p_{1}+p_{2}\right)$ in the associativity constraints. Thus, the transformation $\left(p_{1}, p_{2}, p_{3}\right) \mapsto\left(p_{3}, p_{2}, p_{1}\right)$ is exactly the one that identifies $w(s) w(r)^{s}$ with $w(s r) w(s(1-r) / 1-s r)^{1-s r}$.

We will show in $\$ 9$ below that one can introduce a more refined notion of successor function for thermodynamic semirings, which encodes useful information on the algebraic structure of the semiring, including the lack of associativity, and on the thermodynamical properties of the entropy function. 


\section{The Tsallis entropy}

The Tsallis entropy [52] is a well-studied generalization of Shannon entropy, currently finding application in the statistical mechanics of nonergodic systems, [21]. It is defined by

$$
\operatorname{Ts}_{\alpha}(p)=\frac{1}{\alpha-1}\left(1-p^{\alpha}-(1-p)^{\alpha}\right) .
$$

(A slightly more general form will be analyzed in $\$ 7.1$ below, see (7.2).)

The basic characterizing feature of the Tsallis entropy is the fact that the extensive property (additivity on independent subsystems) typical of the Shannon and Rényi entropies is replaced by a non-extensive behavior. This corresponds, algebraically, to replacing an exponential function (or a logarithm) with an $\alpha$-deformed exponential (or logarithm), see §2.1 of [53], so that the usual Boltzmann principle $S=k \log W$ of statistical mechanics is replaced by its deformed version $S_{\alpha}=k \log _{\alpha} W$, where $\log _{\alpha}(x)=\left(x^{1-\alpha}-1\right)(1-\alpha)$. Thus, instead of additivity $S(A \star B)=S(A)+S(B)$ on the combination of independence systems, one obtains $S_{\alpha}(A \star B)=S_{\alpha}(A)+$ $S_{\alpha}(B)+(1-\alpha) S_{\alpha}(A) S_{\alpha}(B)$. An axiomatic characterization of the Tsallis entropy is described in [20], [49], and [53].

We consider the thermodynamic semiring as in Definition 4.1 with the information measure $S$ given by the Tsallis entropy $S=\mathrm{Ts}_{\alpha}$.

In this case the failure of the associativity condition for the semiring with the $\oplus_{S, T}$ operation is measured by comparing the expressions

$$
\begin{aligned}
& \operatorname{Ts}_{\alpha}\left(p_{1}\right)+\left(1-p_{1}\right) \operatorname{Ts}_{\alpha}\left(\frac{p_{2}}{1-p_{1}}\right) \\
& \quad=\frac{1}{\alpha-1}\left(1-p_{1}^{\alpha}-\left(1-p_{1}\right)^{\alpha}+\frac{p_{2}^{\alpha}}{\left(1-p_{1}\right)^{\alpha-1}}+\frac{\left(1-p_{1}-p_{2}\right)^{\alpha}}{\left(1-p_{1}\right)^{\alpha-1}}\right)
\end{aligned}
$$

and

$$
\begin{aligned}
& \operatorname{Ts}_{\alpha}\left(p_{1}+p_{2}\right)+\left(p_{1}+p_{2}\right) \operatorname{Ts}_{\alpha}\left(\frac{p_{1}}{p_{1}+p_{2}}\right) \\
& =\frac{1}{\alpha-1}\left(1-\left(p_{1}+p_{2}\right)^{\alpha}-\left(1-p_{1}-p_{2}\right)^{\alpha}+\frac{p_{1}^{\alpha}}{\left(p_{1}+p_{2}\right)^{\alpha-1}}+\frac{p_{2}^{\alpha}}{\left(p_{1}+p_{2}\right)^{\alpha-1}}\right) .
\end{aligned}
$$

However, an interesting feature of the Tsallis entropy is that the associativity of the thermodynamic semiring can be restored by a deformation of the operation $\oplus_{S, T}$, depending on the deformation parameter $\alpha$ which makes sense in the previous thermodynamic context, so that the Tsallis entropy becomes the unique function that makes the resulting $\oplus_{S, T, \alpha}$ both commutative and associative.

7.1. A Witt construction for Tsallis entropy. We show here how to deform the thermodynamic semiring structure in a one-parameter family $\oplus_{S, T, \alpha}$ for which $S=$ $\operatorname{Ts}_{\alpha}$ is the only entropy function that satisfies the associativity constraint, along with the commutativity and unity axioms. 
We consider here a slightly more general form of the Tsallis entropy, as the nonassociative information measure the Tsallis entropy [21], defined by

$$
\operatorname{Ts}_{\alpha}(p)=\frac{1}{\phi(\alpha)}\left(p^{\alpha}+(1-p)^{\alpha}-1\right),
$$

where $\alpha \in \mathbb{R}$ is a parameter and $\phi$ is a continuous function such that $\phi(\alpha)(1-\alpha)>0$, whenever $\alpha \neq 1$, with

$$
\lim _{\alpha \rightarrow 1} \phi(\alpha)=0,
$$

and such that there exists $0 \leqslant a<1<b$ with the property that $\phi$ is differentiable on $(a, 1) \cup(1, b)$, and

$$
\lim _{\alpha \rightarrow 1} \frac{d \phi(\alpha)}{d \alpha}<0 .
$$

Note that this implies that the Tsallis entropy reproduces the Shannon entropy in the $\alpha \rightarrow 1$ limit. A typical choice for the normalization is $\phi(\alpha)=1-\alpha$, which reproduces the form (7.1).

Here we work with the more general form (7.2), as we will be able to ensure uniqueness only up to a general $\phi$ satisfying the above requirements.

We find that the Tsallis entropy fits nicely into the context of Witt rings with the following two results.

Theorem 7.1. The Tsallis entropy in the form (7.2) is the unique entropy function that is commutative, has the identity property, and satisfies the $\alpha$-associativity condition

$$
S\left(p_{1}\right)+\left(1-p_{1}\right)^{\alpha} S\left(\frac{p_{2}}{1-p_{1}}\right)=S\left(p_{1}+p_{2}\right)+\left(p_{1}+p_{2}\right)^{\alpha} S\left(\frac{p_{1}}{p_{1}+p_{2}}\right) .
$$

Proof. We assume a priori that $-S$ is concave and continuous. Therefore, $-S$ has a unique maximum, which is positive when $S$ is non-trivial, since $S(0)=0$. Moreover, $S$ is symmetric, so this maximum must occur at $p=1 / 2$. $S$ also has the identity property and the $\alpha$-associativity, so by Suyari [49] and Furuichi [20], this implies $S=\mathrm{Ts}_{\alpha}$, for some $\phi(\alpha)$ satisfying the above properties. The converse follows from direct application of the arguments given in [20] and [49] and is easily verified.

The $\alpha$-associativity condition as one of the characterizing properties for the Tsallis entropy was also discussed in [14].

We can interpret this $\alpha$-associativity as an associativity of an $\alpha$-deformed Witt operation as follows. Fix some $\alpha$ and consider

$$
x \oplus_{S, T, \alpha} y=\sum_{s \in I} e^{T S(s)} x^{s^{\alpha}} y^{(1-s)^{\alpha}} .
$$

We then have the following characterization of associativity.

Theorem 7.2. For $\alpha \neq 0$, the operation $\oplus_{S, T, \alpha}$ is associative if and only if $S$ is $\alpha$-associative, as in (7.3). 
Proof. We find that this operation is associative if and only if

$$
\begin{aligned}
\sum_{s, r \in I} e^{T\left(S(s r)+(1-s r)^{\alpha} S\left(\frac{s(1-r)}{1-s r}\right)\right)} x^{(s r)^{\alpha}} y^{(s(1-r))^{\alpha}} z^{(1-r)^{\alpha}} \\
=\sum_{s, r \in I} e^{T\left(S(s)+s^{\alpha} S(r)\right)} x^{(s r)^{\alpha}} y^{(s(1-r))^{\alpha}} z^{(1-r)^{\alpha}}
\end{aligned}
$$

We make the same substitution as earlier, setting $p_{1}=s r, p_{2}=s(1-r)$, $p_{3}=1-r$. Then the above condition becomes

$$
\begin{aligned}
\sum_{p_{1}+p_{2}+p_{3}=1} e^{T\left(S\left(p_{1}\right)+\left(1-p_{1}\right)^{\alpha} S\left(\frac{p_{2}}{1-p_{1}}\right)\right)} x^{p_{1}^{\alpha}} y^{p_{2}^{\alpha} z_{3}^{\alpha}} & \\
= & \sum_{p_{1}+p_{2}+p_{3}=1} e^{T\left(S\left(p_{1}+p_{2}\right)+\left(p_{1}+p_{2}\right)^{\alpha} S\left(\frac{p_{1}}{p_{1}+p_{2}}\right)\right)} x^{p_{1}^{\alpha}} y^{p_{2}^{\alpha} z_{3}^{\alpha}} .
\end{aligned}
$$

When $\alpha \neq 0$, the map $a \mapsto a^{\alpha}$ is invertible and convex/concave, and the above is a composition of this map with several Legendre transformations, so we can invert this composition to obtain

$$
S\left(p_{1}\right)+\left(1-p_{1}\right)^{\alpha} S\left(\frac{p_{2}}{1-p_{1}}\right)=S\left(p_{1}+p_{2}\right)+\left(p_{1}+p_{2}\right)^{\alpha} S\left(\frac{p_{1}}{p_{1}+p_{2}}\right),
$$

which is exactly the $\alpha$-associativity condition.

It is worth pointing out at this point that in the above deformed Witt construction, we have replaced the energy functional

$$
U=\sum p_{i} E_{i} \quad \text { with } \quad U_{\alpha}=\sum p_{i}^{q} E_{i}
$$

according to our interpretation in 5. In the setting of non-extensive statistical mechanics built upon the Tsallis entropy, this latter expression is exactly the energy functional used. Therefore, the deformed Witt addition is again naturally interpreted as a free energy, now in the more general $q$-deformed thermodynamics.

\section{The Kullback-Leibler divergence}

We now discuss another class of thermodynamic semirings in which both the associativity and the commutativity properties fail, but in which we can encode entropy functions varying over some underlying space or manifold. In particular, we will connect the thermodynamic semirings we consider in this section to the general point of view of information geometry, as developed in [3], [22].

The Kullback-Liebler divergence [27], [28] is a measure of relative entropy, measured by the average logarithmic difference between two probability distributions $p$ and $q$. Since the averaging is done with respect to one of the probability distributions, the KL divergence is not a symmetric function of $p$ and $q$. 
More precisely, the KL divergence of two binary probability distributions $p$ and $q$ is defined by

$$
\mathrm{KL}(p ; q)=p \log \frac{p}{q}+(1-p) \log \frac{1-p}{1-q} .
$$

The negative of the Kullback-Liebler divergence reduces to the Shannon entropy (up to a constant) in the case where $q$ is a uniform distribution. It is also called the information gain, in the sense that it measures the probability law $p$ relative to a given input or reference probability $q$.

We are especially interested here in considering the case where the probability distribution $q$ depends on an underlying space of parameter, continuously or smoothly. Mostly, we will be considering the following two cases.

Definition 8.1. A smooth univariate binary statistical $n$-manifold $\mathcal{Q}$ is a set of binary probability distributions $\mathcal{Q}=(q(\eta))$ smoothly parametrized by $\eta \in \mathbb{R}^{n}$.

A topological univariate binary statistical $n$-space $\mathcal{Q}$ is a set of binary probability distributions $\mathcal{Q}=(q(\eta))$ continuously parameterized by $\eta \in \Xi$, with $\Xi$ a compact Hausdorff topological space.

The first case leads to the setting of information geometry [3], [22], while the second case is more suitable for treating multifractal systems [6].

We then consider thermodynamic semiring in the more general form of Definition 4.4. Let $\mathcal{X}$ be either a compact subset of $\mathbb{R}^{n}$ in the case of a smooth univariate binary statistical manifold or a closed subset of a compact Hausdorff space $\Xi$ in the topological case of Definition 8.1. We consider the space of continuous functions $\mathcal{R}=C(\mathcal{X}, R)$, where the semiring $K$ is either $\mathbb{R}^{\min ,+} \cup\{\infty\}$ or $\mathbb{R}_{\geq 0}^{\max , *}$, or in the smooth case we take $\mathcal{R}=C^{\infty}(\mathcal{X}, K)$.

Given $q=q(\eta)$ in $\mathcal{Q}$, we can endow the space $\mathcal{R}$ of functions with a thermodynamic semiring structure as in Definition 4.4, where the deformed addition operation is given by

$$
x(\eta) \oplus_{\mathrm{KL}_{\eta}, \rho} y(\eta)=\sum_{p \in \mathbb{Q} \cap[0,1]} \rho^{-\mathrm{KL}(p ; q(\eta))} x(\eta)^{p} y(\eta)^{1-p},
$$

where $\rho$ is the parameter of the deformation. Note we use the negative of the KL divergence because we are interested in it as a measure of relative entropy, rather than relative information, concepts often conceptually distinct but always related by a minus sign.

In the case when $q(\eta) \equiv 1 / 2$ is uniform for all $\eta$, we get back the original case with the Shannon entropy up to a shift factor

$$
\begin{aligned}
\left.x \oplus_{\mathrm{KL}_{\eta}, \rho} y\right|_{q(\eta) \equiv 1 / 2} & =\max _{p}\left(-\rho\left(p \log \frac{p}{1 / 2}+(1-p) \log \frac{1-p}{1 / 2}\right)+p x+(1-p) y\right) \\
& =\max _{p}(\rho \operatorname{Sh}(p)+p x+(1-p) y)+\rho \log 2 .
\end{aligned}
$$


We note that we can calculate this operation explicitly over $\mathbb{R}^{\min ,+} \cup\{\infty\}$ and $\mathbb{R}_{\geq 0}^{\max , *}$. We obtain the following result by arguing as in Proposition 4.3.

Proposition 8.2. We have the following expression over $\mathbb{R}^{\mathrm{min},+} \cup\{\infty\}$

$$
x \oplus_{\mathrm{KL}} y=-T \log \left(e^{-\frac{x}{q T}}+e^{-\frac{y}{(1-q) T}}\right)
$$

and the following expression over $\mathbb{R}_{\geq 0}^{\max , *}$.

$$
x \oplus_{\mathrm{KL}} y=\left(\left(\frac{x}{q}\right)^{1 / T}+\left(\frac{y}{1-q}\right)^{1 / T}\right)^{T}
$$

The first observation then is that the additive structures (8.1) are in general not commutative.

Proposition 8.3. The thermodynamic semiring structure

$$
x \oplus_{\mathrm{KL}} y=\sum_{p \in \mathbb{Q} \cap[0,1]} \rho^{-\mathrm{KL}(p ; q)} x^{p} y^{1-p}
$$

is commutative if and only if $q=1 / 2$. The lack of commutativity is measured by the transformation $q \mapsto 1-q$.

Proof. This is immediate from the previous calculation, but we perform the proof over general $K$. We find that

$$
\mathrm{KL}(1-p ; q)=(1-p) \log \frac{1-p}{q}+p \log \frac{p}{1-q} .
$$

This is related to $\operatorname{KL}(p ; q)$ by the transformation $q \mapsto 1-q$. Thus, $\operatorname{KL}(p ; q)=$ $\mathrm{KL}(1-p ; q)$ when $\log \frac{1-q}{q}=0$, that is, when $q=1 / 2$. This is exactly when the Shannon entropy case is reproduced, so the only case when the addition (8.3) based on the Kullback-Liebler divergence is commutative is when it agrees with the Shannon entropy up to a shift factor.

For the associativity condition we find the following result.

Proposition 8.4. The lack of associativity of the thermodynamic semiring (8.3) is measured by the transformation $\left(p_{1}, p_{2}, p_{3} ; q\right) \mapsto\left(p_{3}, p_{2}, p_{1} ; 1-q\right)$.

Proof. Again we proceed over general $K$. We have

$$
\begin{aligned}
\mathrm{KL}\left(p_{1} ; q\right) & +\left(1-p_{1}\right) \operatorname{KL}\left(\frac{p_{2}}{1-p_{1}} ; q\right) \\
= & p_{1} \log \frac{p_{1}}{q}+\left(1-p_{1}\right) \log \frac{1-p_{1}}{1-q}+p_{2} \log \frac{p_{2}}{\left(1-p_{1}\right) q} \\
& +\left(1-p_{1}-p_{2}\right) \log \frac{1-p_{1}-p_{2}}{\left(1-p_{1}\right)(1-q)}
\end{aligned}
$$




$$
\begin{aligned}
= & p_{1} \log \frac{p_{1}}{q}+\left(1-p_{1}\right) \log \frac{1-p_{1}}{1-q}+p_{3} \log \frac{p_{3}}{1-q} \\
& +p_{2} \log \frac{p_{2}}{q}-\left(1-p_{1}\right) \log \left(1-p_{1}\right),
\end{aligned}
$$

while

$$
\begin{aligned}
\mathrm{KL}\left(p_{1}\right. & \left.+p_{2} ; q\right)+\left(p_{1}+p_{2}\right) \operatorname{KL}\left(\frac{p_{1}}{p_{1}+p_{2}} ; q\right) \\
= & \left(p_{1}+p_{2}\right) \log \frac{p_{1}+p_{2}}{q}+\left(1-p_{1}-p_{2}\right) \log \frac{1-p_{1}-p_{2}}{1-q} \\
& +\left(p_{1}+p_{2}\right) \frac{p_{1}}{p_{1}+p_{2}} \log \frac{p_{1}}{\left(p_{1}+p_{2}\right) q} \\
& +\left(p_{1}+p_{2}\right) \frac{p_{2}}{p_{1}+p_{2}} \log \frac{p_{2}}{\left(p_{1}+p_{2}\right)(1-q)} \\
= & \left(p_{1}+p_{2}\right) \log \frac{p_{1}+p_{2}}{q}+\left(1-p_{1}-p_{2}\right) \log \frac{1-p_{1}-p_{2}}{1-q}+p_{1} \log \frac{p_{1}}{q} \\
& +p_{2} \log \frac{p_{2}}{1-q}-\left(p_{1}+p_{2}\right) \log \left(p_{1}+p_{2}\right) \\
= & p_{3} \log \frac{p_{3}}{1-q}+\left(1-p_{3}\right) \log \frac{1-p_{3}}{q}+p_{1} \log \frac{p_{1}}{q} \\
& +p_{2} \log \frac{p_{2}}{1-q}-\left(1-p_{3}\right) \log \left(1-p_{3}\right) .
\end{aligned}
$$

These are related by the transformation $\left(p_{1}, p_{2}, p_{3} ; q\right) \mapsto\left(p_{3}, p_{2}, p_{1} ; 1-q\right)$.

Notice that, because of the presence of the shift in (8.2) with respect to the Shannon entropy, in the case $q=1 / 2$ we find

$$
\begin{aligned}
\mathrm{KL} & \left(p_{1} ; \frac{1}{2}\right)+\left(1-p_{1}\right) \mathrm{KL}\left(\frac{p_{2}}{1-p_{1}} ; \frac{1}{2}\right) \\
& =p_{1} \log p_{1}+p_{2} \log p_{2}+p_{3} \log p_{3}+\log 2+\left(1-p_{1}\right) \log 2
\end{aligned}
$$

while

$$
\begin{aligned}
\mathrm{KL} & \left(p_{1}+p_{2} ; \frac{1}{2}\right)+\left(p_{1}+p_{2}\right) \mathrm{KL}\left(\frac{p_{1}}{p_{1}+p_{2}} ; \frac{1}{2}\right) \\
& =p_{1} \log p_{1}+p_{2} \log p_{2}+p_{3} \log p_{3}+\log 2+\left(1-p_{3}\right) \log 2 .
\end{aligned}
$$

Thus, associativity is not automatically obtained in the uniform distribution case, but instead we have associativity up to a shift.

By Proposition 8.4 we see that, in the case of a thermodynamic semiring $\mathcal{R}=$ $C(\mathcal{X}, K)$ or $\mathcal{R}=C^{\infty}(\mathcal{X}, K)$, for a topological or smooth univariate binary statistical space, if one can find an involution $\alpha: \mathcal{X} \rightarrow \mathcal{X}$ of the parameter space such that 
$q(\alpha(\eta))=1-q(\eta)$, then one can consider the transformation $x(\eta) \mapsto x(\alpha(\eta))$ and one finds that

$$
x(\eta) \oplus_{\mathrm{KL}_{q(\eta)}} y(\eta)=y(\alpha(\eta)) \oplus_{\mathrm{KL}_{q(\alpha(\eta))}} x(\alpha(\eta)) .
$$

Moreover, the morphism

$$
A:(x(\eta), y(\eta), z(\eta)) \mapsto(z(\alpha(\eta)), y(\alpha(\eta)), x(\alpha(\eta)))
$$

measures the lack of associativity by making the diagram

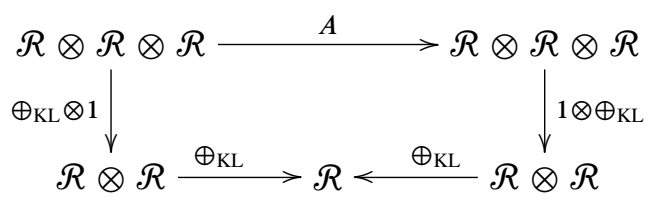

commute.

8.1. Applications to multifractal systems. Consider the case of a Cantor set $\mathcal{X}$ identified, through its symbolic dynamics interpretation, as the one sided full shift space $\Sigma_{2}^{+}$on the alphabet $\{0,1\}$, see $\S 1.3$ of [42].

For $\eta \in \mathcal{X}$, let $a_{n}(\eta)$ denote the number of 1's that appear in the first $n$ digits $\eta_{1}, \ldots, \eta_{n}$ of $\eta$. We set

$$
q(\eta)=\lim _{n \rightarrow \infty} \frac{a_{n}(\eta)}{n}
$$

if this limit exists. We denote by $y \subset \mathcal{X}$ the set of points for which the limit (8.4) exists.

The limit (8.4) determines several important dynamical properties related to the fractal geometry of $\mathcal{X}$. For example, suppose that $\mathcal{X}$ is a uniform Cantor set obtained from a contraction map $f$ with contraction ratio $\lambda$, endowed with a Bernoulli measure $\mu_{p}$ for a given $0<p<1$, defined by assigning measure

$$
\mu_{p}\left(\mathcal{X}\left(w_{1}, \ldots, w_{n}\right)\right)=p^{a_{n}(w)}(1-p)^{n-a_{n}(w)}
$$

to the cylinder sets

$$
\mathcal{X}\left(w_{1}, \ldots, w_{n}\right)=\left\{\eta \in \mathcal{X} \mid \eta_{i}=w_{i}, i=1, \ldots, n\right\} .
$$

Then the local dimension of $\mathcal{X}$ at a point $\eta \in \mathcal{Y}$ is given by ( $\$ 4.17$ of [42])

$$
d_{\mu_{p}}(\eta)=\frac{q(\eta) \log p+(1-q(\eta)) \log (1-p)}{\log \lambda}
$$

while the local entropy of the map $f$ is given by ( $\$ 4.18$ of [42])

$$
h_{\mu_{p}, f}(\eta)=q(\eta) \log p+(1-q(\eta)) \log (1-p) .
$$


For a non-uniform Cantor set $\mathcal{X}$ with two contraction ratios $\lambda_{1}$ and $\lambda_{2}$ on the two intervals, the Lyapunov exponent of $f$ is given by ( $\$ 4.20$ of [42])

$$
\lambda_{f}(\eta)=q(\eta) \log \lambda_{1}+(1-q(\eta)) \log \lambda_{2} .
$$

One knows that, given a Bernoulli measure $\mu_{p}$ on the Cantor set $\mathcal{X}$, there is a set $\mathcal{Z} \subset \mathcal{X}$ of full measure $\mu_{p}(\mathcal{Z})=1$, for which $q(\eta)=p$ (Proposition 4.5 of [42]). The choice of the uniform measure $\mu_{1 / 2}$ yields a full measure subset $Z_{1 / 2}$ on which the limit $q(\eta)=1 / 2$ is the uniform distribution (the fair coin case). In general one can stratify the set $y \subset \mathcal{X}$ into level sets of $q(\eta)$. This provides a decomposition of the Cantor set as a multifractal.

Looking at this setting from the point of view of thermodynamic semirings suggests considering the set of functions $C(\mathcal{Y}, K)$ endowed with the pointwise operation $\oplus_{\mathrm{KL}_{q(\eta)}, T}$, with the Kullback-Leibler divergence $\mathrm{KL}(p ; q(\eta))$, for $q(\eta)$ defined as in (8.4). Then we see that, without the need to choose a measure on $\mathcal{X}$, the algebraic properties of the thermodynamic semiring automatically select the "fair coin subfractal" $Z_{1 / 2}$.

Proposition 8.5. For $\mathcal{Z} \subset \mathcal{Y}$, the semiring $C(\mathcal{Z}, K)$, with the operation $\bigoplus_{\mathrm{KL}_{q(\eta)}, T}$, for $q(\eta)$ as in (8.4), is commutative if and only if $\mathbb{Z} \subset \mathbb{Z}_{1 / 2}$ is a "fair coin" subset.

Proof. This follows immediately from Proposition 8.3.

Moreover, we can see geometrically the involution that measures the lack of commutativity as in Proposition 8.3 and the lack of associativity as in Proposition 8.4.

Proposition 8.6. The homeomorphism $\gamma: \mathcal{X} \rightarrow \mathcal{X}$ given by the involution that exchanges $0 \leftrightarrow 1$ in the digits of $\eta$ in the shift space $\Sigma_{2}^{+}$implements the involution $q(\eta) \mapsto 1-q(\eta)$ that measures the lack of commutativity and that, together with the involution $\left(p_{1}, p_{2}, p_{3}\right) \mapsto\left(p_{3}, p_{2}, p_{1}\right)$ also measures the lack of associativity. Thus, the morphism $x(\eta) \mapsto x(\gamma(\eta))$ restores commutativity in the sense that

$$
x(\eta) \oplus_{\mathrm{KL}_{q(\eta)}} y(\eta)=y(\gamma(\eta)) \oplus_{\mathrm{KL}_{q(\gamma(\eta)}} x(\gamma(\eta)),
$$

while $A: \mathcal{R} \otimes \mathcal{R} \otimes \mathcal{R} \rightarrow \mathcal{R} \otimes \mathcal{R} \otimes \mathcal{R}$ given by

$$
A(x(\eta), y(\eta), z(\eta))=(z(\gamma(\eta)), y(\gamma(\eta)), x(\gamma(\eta)))
$$

restores associativity, making the diagram commute

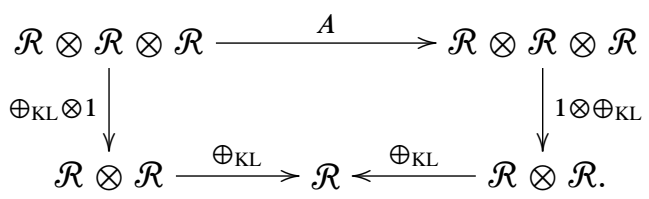

Proof. This follows immediately from Proposition 8.3 and Proposition 8.4 , by observing that the $q(\eta)$ defined as in (8.4) satisfies $q(\gamma(\eta))=1-q(\eta)$ since $a_{n}(\gamma(\eta))=$ $n-a_{n}(\eta)$ for all $\eta \in \mathcal{X}$. 
8.2. Multivariate binary statistical manifolds. We see that in the univariate case, the extremal $p$ value is the unique probability distribution minimizing the KLdivergence to $q$ subject to the soft constraint coming from the energy functional $p x+(1-p) y$, see $\S 5$. This is important because minimizing the KL divergence is maximizing likelihood, and this plays an important role in marginal estimation, belief propagation, mutual information calculation, see [22] and [3].

A more interesting case is that of multivariate statistical manifolds. To maintain the same features as in the univariate case, we will find that a hyperring structure is most natural. See [54], [11] for an introduction and relevant facts of hyperstructures. We first note the following fact.

Proposition 8.7. If $p$ and $q$ are two distributions, we denote by $p_{i}$ and $q_{i}$ their $i$-th marginal distribution. Then $\mathrm{KL}(p ; q)=\sum_{i} \operatorname{KL}\left(p_{i} ; q_{i}\right)$.

Proof. We have

$$
\begin{aligned}
\mathrm{KL}(p ; q)= & p_{1} \ldots p_{n} \log \frac{p_{1} \ldots p_{n}}{q_{1} \ldots q_{n}}+\left(1-p_{1}\right) p_{2} \ldots p_{n} \log \frac{\left(1-p_{1}\right) p_{2} \ldots p_{n}}{\left(1-q_{1}\right) q_{2} \ldots q_{n}}+\cdots \\
& \ldots+\left(1-p_{1}\right) \ldots\left(1-p_{n}\right) \log \frac{\left(1-p_{1}\right) \ldots\left(1-p_{n}\right)}{\left(1-q_{1}\right) \ldots\left(1-q_{n}\right)} \\
= & p_{1} \ldots p_{n}\left(\log \frac{p_{1}}{q_{1}}+\cdots+\log \frac{p_{n}}{q_{n}}\right)+\cdots+\left(1-p_{1}\right) \ldots \\
& \ldots\left(1-p_{n}\right)\left(\log \frac{1-p_{1}}{1-q_{1}}+\cdots+\log \frac{1-p_{n}}{1-q_{n}}\right) \\
= & p_{1} \log \frac{p_{1}}{q_{1}}\left(p_{2} \ldots p_{n}+\left(1-p_{2}\right) \ldots p_{n}+\cdots\right) \\
& +\left(1-p_{1}\right) \log \frac{1-p_{1}}{1-q_{1}}\left(p_{2} \ldots p_{n}+\cdots\right)+\cdots \\
= & p_{1} \log \frac{p_{1}}{q_{1}}\left(\left(1+p_{2}-p_{2}\right)\left(p_{3} \ldots p_{n}+\ldots\right)\right)+\cdots \\
= & p_{1} \log \frac{p_{1}}{q_{1}}+\left(1-p_{1}\right) \log \frac{1-p_{1}}{1-q_{1}}+\cdots+\left(1-p_{n}\right) \log \frac{1-p_{n}}{1-q_{n}} \\
= & \sum_{i} \operatorname{KL}\left(p_{i} ; q_{i}\right) .
\end{aligned}
$$

Thus, if we can ensure that the sum of the KL divergences of the marginal distributions is minimized, then the total KL divergence will be minimized.

8.3. Product of semirings and hyperfield structure. We proceed by taking the semiring

$$
\mathcal{R}=C(\{1, \ldots, n\}, K)=K^{\otimes n} .
$$


It is tempting to define the operations on $\mathcal{R}$ coordinate-wise, however, since we want to consider an $n$-ary probability distribution and not $n$ binary probability distributions, there should be some dependence between coordinates that takes advantage of the previous proposition. In short, we would like to put an ordering on $\mathcal{R}$ that ensures the trace

$$
\left(x_{1}, \ldots, x_{n}\right) \rightarrow x_{1}+\cdots+x_{n} \in K
$$

is maximized. This ordering does not uniquely determine a maximum between two tuples. We thus for sake well-definedness of the addition on $K$ and define $\left(x_{1}, \ldots, x_{n}\right)+\left(y_{1}, \ldots, y_{n}\right)$ to be the set of tuples $\left(z_{1}, \ldots, z_{n}\right)$ with $z_{i}=x_{i}$ or $y_{i}$ that maximize $z_{1}+\cdots+z_{n}$ in the ordering on $K$. This, together with coordinate-wise multiplication defines a characteristic one hyperfield structure on $\mathcal{R}$. We then define the Witt operation for some information measures $S_{1}, \ldots, S_{n}$ over $K=\mathbb{R}^{\min ,+} \cup\{\infty\}$ by

$$
\begin{aligned}
& x \oplus_{S_{1}, \ldots, S_{n} y} \\
& \quad=\min _{p_{1}, \ldots, p_{n}}\left(p_{1} x_{1}+\left(1-p_{1}\right) y_{1}-T S_{1}\left(p_{1}\right), \ldots, p_{n} x_{n}+\left(1-p_{n}\right) y_{n}-T S_{n}\left(p_{n}\right)\right),
\end{aligned}
$$

where $x=\left(x_{1}, \ldots, x_{n}\right), y=\left(y_{1}, \ldots, y_{n}\right)$, now we consider the $p_{i}$ as marginal probabilities, and the min operation is the multivalued hyperring addition. When each $S_{i}$ is the KL-divergence from some $q_{i}$, by the previous proposition, the results of this operation are exactly the distributions with marginal probabilities $\left(p_{1}, \ldots, p_{n}\right)$ minimizing the KL-divergence to the marginal probabilities $\left(q_{1}, \ldots, q_{n}\right)$ subject to the soft constraint coming from the energy functional

$$
U=\sum p_{i} x_{i}+\left(1-p_{i}\right) y_{i} .
$$

The lack of well-definedness of this addition can be interpreted in the thermodynamic context as the non-uniqueness of equilibria, via the existence of meta-equilibrium states. Indeed, when the $q_{i}$ describe a uniform distribution, we find that this addition is in fact well defined.

Note that these hyperfields are slightly different from those considered in [54]. However, just as taking $T \rightarrow 0$ for the Shannon entropy semiring reproduces the "dequantized" tropical semiring, we can take $T \rightarrow 0$ for the KL divergence semiring to get a "dequantized" tropical hyperfield. This reproduces the undeformed addition defined on $K$ above. Note that this is not the same as Oleg Viro's tropical hyperfield discussed in [54].

We can encode more information about a space in the ring deformation by restricting the marginal probabilities we sum over, in particular we can restrict the minimizing process to certain submanifolds of our probability manifold such as the e-flat or m-flat manifolds typically considered in [3], since the KL-divergence is related to the Fisher information matrix defining the Riemannian structure. See also the comments in $\S 11.1$ below. 


\section{The successor in thermodynamic semirings}

Given a thermodynamic semiring in the sense of Definition 4.1, we let

$$
\lambda(x, T)=x \oplus_{S} 0 \equiv \min _{p}(p x-T S(p)) .
$$

Then $\lambda: K \times \mathbb{R} \rightarrow K$ is the Legendre transform of $T S:[0,1] \rightarrow \mathbb{R}$. If we assume that $S$ has a unique maximum, then we can invert the Legendre transform, so that

$$
T S(p)=\min _{x}(p x-\lambda(x, T)) .
$$

Therefore, when $S$ is concave/convex, we can recover it from the semiring. We call $\lambda$ the successor function since 0 is the multiplicative identity, and over general $K$ we can write $\lambda(x, T)=x \oplus_{S} 1$. When multiplication distributes over addition, we can write

$$
x \oplus_{S} y=\lambda(x-y, T)+y .
$$

We will tend to suppress the $T$ dependence of $\lambda$. Each of the algebraic properties of $S$ and $K$ translate into the language of $\lambda$.

Proposition 9.1. The entropy function $S$ has the following properties.

(1) It satisfies the commutativity axiom $S(p)=S(1-p)$ (hence $\oplus_{S, T}$ is commutative) if and only if

$$
\lambda(x)-\lambda(-x)=x .
$$

(2) It satisfies the left identity axiom $S(0)=0$ (hence $\oplus_{S}$ has left identity $\infty$ ) if and only if $\lambda(x) \leqslant 0$ and $\lim _{x \rightarrow \infty} \lambda(x)=0$.

(3) It satisfies the right identity axiom $S(1)=0$ (hence $\oplus_{S}$ has left identity $\infty$ ) if and only if $\lambda(x) \leqslant x$ and $\lambda(x) \sim x$, as $x \rightarrow-\infty$.

(4) It satisfies the associativity constraint making $\oplus_{S}$ associative iff

$$
\lambda(x-\lambda(y))+\lambda(y)=\lambda(\lambda(x-y)+y) .
$$

Proof. Facts (1) and (4) are immediate from the definition. The properties (2) and (3) arise from the fact that $\lambda$ should be continuous at $\infty$ and $-\infty$. We then read $\infty \oplus_{S} x$ and $x \oplus_{S} \infty$ as $\lim _{y \rightarrow \infty} y \oplus_{S} x$ and $\lim _{y \rightarrow \infty} x \oplus_{S} y$, respectively. Each of these should equal $x$, and in terms of $\lambda$ we see that $\lim _{y \rightarrow \infty} y \oplus_{S} x=\lim _{y \rightarrow \infty} \lambda(y-x)+x$ and $\lim _{y \rightarrow \infty} x \oplus_{S, T} y=\lim _{y \rightarrow \infty} \lambda(x-y)+y$, thus proving (2) and (3).

In the case of the Shannon entropy $S=\mathrm{Sh}$ and KL-divergence $S=-\mathrm{KL}(p ; q)$, we have the following forms for the successor function.

Proposition 9.2. For Shannon entropy,

$$
\lambda^{\mathrm{Sh}}(x, T)=-T \log \left(1+e^{-x / T}\right)
$$


over $\mathbb{R}^{\min ,+} \cup\{\infty\}$, and

$$
\lambda^{\mathrm{Sh}}(x, T)=\left(1+x^{1 / T}\right)^{T}
$$

over $\mathbb{R}_{\geqslant 0}^{\max ,+}$. For the KL-divergence,

$$
\lambda^{\mathrm{KL}}(x, T)=-T \log \left(1+e^{-x / q T}\right)
$$

over $\mathbb{R}^{\min ,+} \cup\{\infty\}$, and

$$
\lambda^{\mathrm{KL}}(x, T)=\left(1 /(1-q)^{1 / T}+(x / q)^{1 / T}\right)^{T}
$$

over $\mathbb{R}_{\geqslant 0}^{\max ,+}$.

Proof. This follows directly from the definition of $\lambda(x, T)=x \oplus_{S} 0$, and the isomorphism $-\log$ relating the semirings $\mathbb{R}_{\geq 0}^{\max , *}$ and $\mathbb{R}^{\min ,+} \cup\{\infty\}$.

Figures 1, 2 and 3 show examples of a plot of $\lambda^{\text {Sh }}$ plotted versus $x$, for different values of $T$.

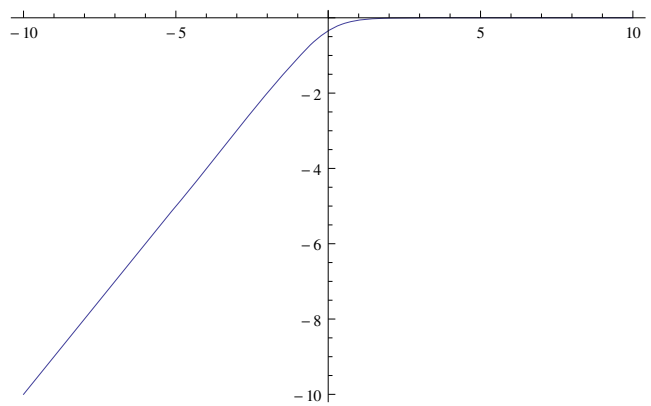

Figure 1. The successor function $\lambda^{\text {Sh }}$ for $T=0.5$.

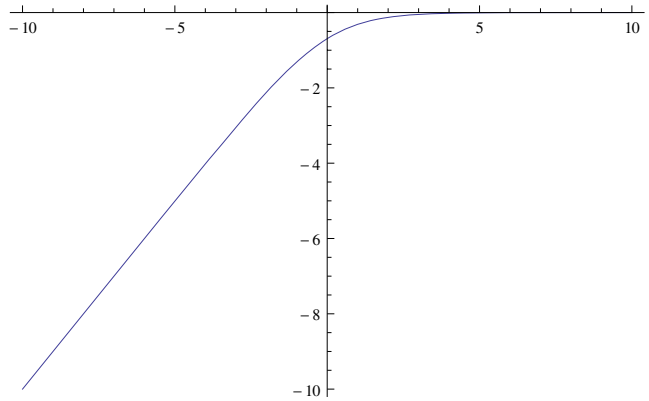

Figure 2. The successor function $\lambda^{\text {Sh }}$ for $T=1$. 


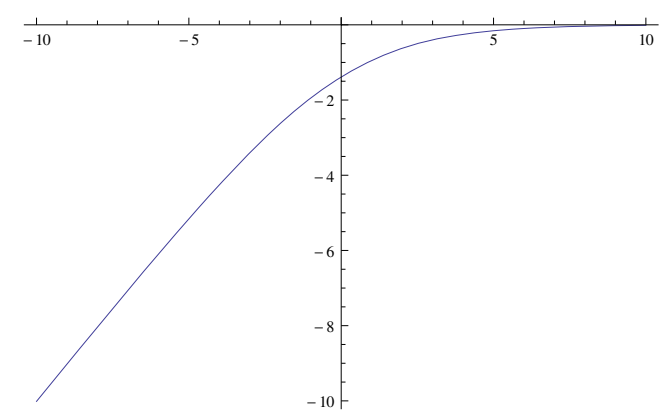

Figure 3. The successor function $\lambda^{\text {Sh }}$ for $T=2$.

9.1. Successor function for Tsallis entropy. Consider now the case of the Tsallis entropy

$$
\operatorname{Ts}_{\alpha}(p)=\frac{1}{1-\alpha}\left(p^{\alpha}+(1-p)^{\alpha}-1\right) .
$$

Proposition 9.3. The successor function $\lambda^{\mathrm{Ts} \alpha}(x, T)$ for the Tsallis entropy is given by

$$
\lambda^{\operatorname{Ts}_{\alpha}}(x, T)= \begin{cases}0 & \left|\frac{\alpha}{1-\alpha}\right|<x / T, \\ g(x) & -\left|\frac{\alpha T}{1-\alpha}\right|<x / T<\left|\frac{\alpha}{1-\alpha}\right|, \\ x & x / T<-\left|\frac{\alpha T}{1-\alpha}\right|,\end{cases}
$$

where $g(x)$ is given by applying Ts to the inverse of its derivative.

Proof. We have

$$
\frac{\partial \mathrm{Ts}}{\partial p}=\frac{\alpha}{1-\alpha}\left(p^{\alpha-1}-(1-p)^{\alpha-1}\right) .
$$

We see the derivative of $\operatorname{Ts}_{\alpha}$ has range $\left[-\left|\frac{\alpha}{1-\alpha}\right|,\left|\frac{\alpha}{1-\alpha}\right|\right]$, so that we obtain (9.1).

Figure 4 shows an example of a plot of $\lambda^{\mathrm{Ts}_{\alpha}}$ plotted versus $x$. In the limit $\alpha \rightarrow \infty$, one has $\operatorname{Ts}_{\alpha}=\chi_{(0,1]}$, so indeed $\lambda^{\mathrm{Ts}_{\infty}}(x)=x \chi_{[-\infty, 0)}(x)$ for finite temperature. When $\alpha<0, \operatorname{Ts}_{\alpha}$ is convex, so $\lambda$ becomes concave in this region, as expected.

9.2. Successor function for Rényi entropy. We now consider again the Réyni entropy given by

$$
\operatorname{Ry}_{\alpha}(p)=\frac{1}{1-\alpha} \log \left(p^{\alpha}+(1-p)^{\alpha}\right) .
$$

We have

$$
\frac{\partial \mathrm{Ry}}{\partial p}=\frac{\alpha}{1-\alpha}\left(p^{\alpha-1}+(1-p)^{\alpha-1}\right) /\left(p^{\alpha}+(1-p)^{\alpha}\right) .
$$

This time, however, the derivative has range $\mathbb{R}$, so that we have both $\lambda^{\operatorname{Ry}_{\alpha}}(x)<x$ and $\lambda^{\operatorname{Ry}_{\alpha}}(x)<0$. 


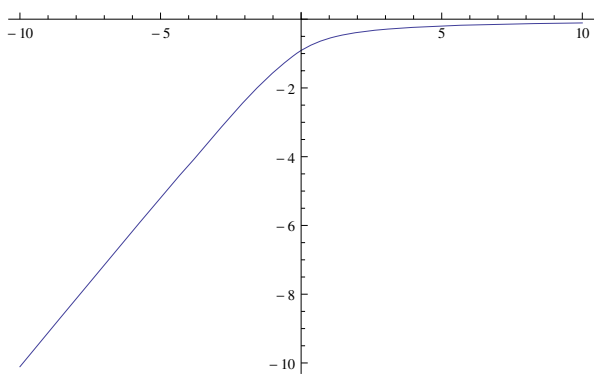

Figure 4. The successor function $\lambda_{\alpha}^{\mathrm{Ts}}$ for $\alpha=0.5$ and $T=1$.

Figures 5 and 6 show examples of a plot of $\lambda^{\mathrm{Ry}} \mathrm{y}_{\alpha}$ plotted versus $x$, for different values of $\alpha$.

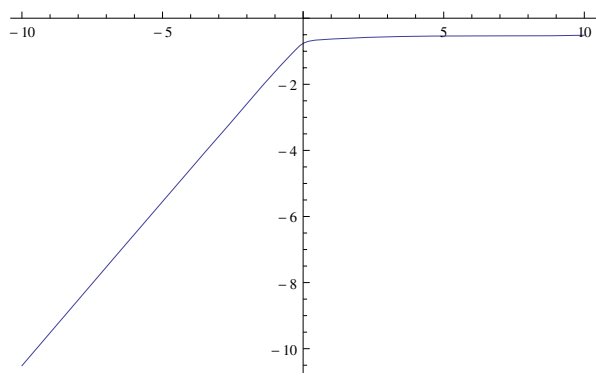

Figure 5. The successor function $\lambda^{\mathrm{Ry}}$ for $\alpha=0.1$ and $T=1$.

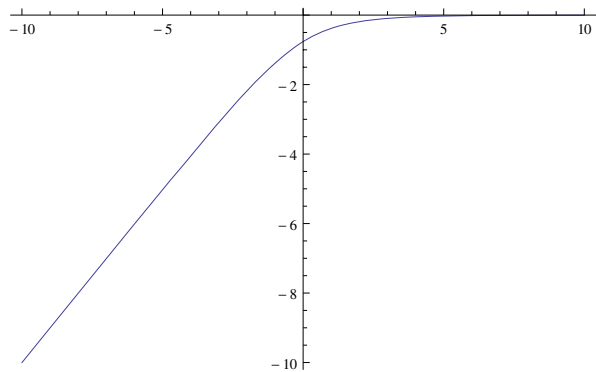

Figure 6. The successor function $\lambda^{\mathrm{Ry}}$ for $\alpha=0.9$ and $T=1$.

One can see, by comparing these various graphs for the different entropy functions that increasing $T$ has the effect of smoothing the transition, while increasing $\alpha$ sharpens it. 
9.3. Cumulants generating function. In this section we give a thermodynamical interpretation of the successor function.

Recall that, for a random variable $X$, if $M_{X}(t)$ denotes the generating function for the momenta of $X$,

$$
M_{X}(t)=\langle\exp (t X)\rangle=\sum_{m=0}^{\infty} \mu_{m} \frac{t^{m}}{m !},
$$

then the cumulants $\left\{\kappa_{n}\right\}$ of $X$ are defined as the coefficients of the power series expansion of the function $\log M_{X}(t)$,

$$
\log M_{X}(t)=\sum_{n=0}^{\infty} \kappa_{n} \frac{t^{n}}{n !} .
$$

The information contained in cumulants or momenta is equivalent, though cumulants have the advantage that they behave additively over independent variables.

We then have the following result. We consider the case of an analytic $\lambda$, which is reasonable when attempting to gain a thermodynamic understanding, as the microscopic dynamics are usually assumed to be analytic.

Proposition 9.4. Let $\lambda(x, T)$ be the successor function of a thermodynamic semiring $K$. Assume that $\lambda(x, T)$ is analytic. Then the function $-\lambda(x, T) / T$ is the cumulant generating function of the probability distribution for the energy $E$, in the variable $-1 / T=-\beta$. Namely, if we write the $n$-th cumulant as $\kappa_{n}=\left\langle E^{n}\right\rangle_{c}$, we have

$$
(-1)^{n+1} \frac{\partial^{n}}{\partial \beta^{n}}(\beta \lambda(x, T))=\left\langle E^{n}\right\rangle_{c} .
$$

Proof. In thermodynamics, $Z(\beta)=\langle\exp (-\beta E)\rangle$ is the partition function, where $\beta=1 / T$ is the inverse temperature and $E$ is the energy. The Helmholtz free energy is then given by

$$
F=-T \log \langle\exp (-E / T)\rangle .
$$

Up to a factor of $-1 / T$, the Helmholtz free energy is in fact the cumulant generating function for the random variable given by the energy $E$. As observed already in $\S 5$, the Helmholtz free energy is the Legendre transform of the entropy, and can therefore we identified, again up to a factor of $-1 / T$, with the function $\lambda(x, T)$.

We can of course perform this proof without reference to the thermodynamics. That is to say: the Legendre transform structure of the whole ordeal is independent of the information measure we select as long as we select one which is concave and analytic.

In particular, from (9.2) we have

$$
\lambda(x, T)-T \frac{\partial}{\partial T} \lambda(T, x)=\langle E\rangle=p_{\text {eq }} x,
$$


where $p_{\mathrm{eq}}=p_{T}(x)$ is equilibrium value of the mole fraction. We know that $\lambda(x, T)=\min _{p}(p x-T S(p))=p_{T}(x)-T S\left(p_{T}(x)\right)$. We see that $p_{T}(x)$ satisfies

$$
x / T=\frac{d}{d p} S\left(p_{T}(x)\right),
$$

so we can write $p_{T}(x)=p(x / T)$ and $\lambda(T, x)=\lambda(x / T)$. Notice that this explains the effect that changing the temperature has on $\oplus S, T$.

From the definition, we calculate

$$
\frac{\partial}{\partial T} \lambda(x / T)=x \frac{\partial}{\partial T} p(x / T)-S(p(x / T))-T \frac{\partial}{\partial T} p(x / T) \frac{d}{d p} S(p(x / T)),
$$

which, by the above property, is just $-S(p(x / T))$, proving the above relation. Note this holds for arbitrary smooth, concave entropy functions. Similarly, we calculate

$$
\frac{\partial}{\partial x} \lambda(x / T)=x p(x / T),
$$

so that

$$
\lambda(x / T)=x \frac{\partial}{\partial x} \lambda(x / T)+T \frac{\partial}{\partial T} \lambda(x / T) .
$$

This is a well-known property of the Legendre transform of smooth functions.

\section{Entropy operad}

A categorical and operadic point of view on convex spaces and entropy functions was recently proposed in [4], [5], [18], [19]. Here we will use a similar viewpoint to describe generalized associativity conditions on thermodynamic semirings.

More precisely, we consider here the more general question of how binary (or more complicated) information measures can be built up to ones for $n$-ary random variables for any $n \geqslant 2$. This will give us some interesting correspondences between the combinatorics of such "guessing games" and generalized associativity conditions in an operad with $n$-ary operations defined over $K$ like $x_{1} \oplus_{S} \cdots \oplus_{S} x_{n}$ with some choice of parenthesizing. In this section, we will assume for simplicity that $K$ is $\mathbb{R}^{\min ,+} \cup\{\infty\}$, although, once again, this is only a notational convention chosen to elucidate certain expressions. All the statements made here could be translated into the greater generality for real characteristic one semifields.

Operads were first introduced in [38] in the theory of iterated loop spaces and have since seen a broad range of applications in algebra, topology, and geometry. We recall briefly some basic facts about operads that we will need later, see [39].

An operad is a collection of objects from a symmetric monoidal category $S$ with product $\otimes$ and unit object $\kappa$. In particular, for each $j \in \mathbb{N}$, we have an object $\ell(j)$, thought of as parameter objects for $j$-ary operations, with actions by the symmetric 
group $\mathrm{Sym}_{j}$, thought of as permuting inputs. We also have a unit map $\eta: \kappa \rightarrow \ell(1)$ and composition maps

$$
\gamma: \mathscr{C}(k) \otimes \mathscr{C}\left(j_{1}\right) \otimes \cdots \otimes \mathscr{C}\left(j_{k}\right) \rightarrow \mathscr{C}\left(j_{1}+\cdots+j_{k}\right)
$$

which are suitably associative, unital, and equivariant under the action of $\operatorname{Sym}_{k}$ such that if $\sigma \in \operatorname{Sym}_{k}$, then

$$
\gamma\left(c_{k} \otimes c_{\sigma\left(j_{1}\right)} \otimes \cdots \otimes c_{\sigma\left(j_{k}\right)}\right)=\gamma\left(\sigma\left(c_{k}\right) \otimes c_{j_{1}} \otimes \cdots \otimes c_{j_{k}}\right) .
$$

A $\mathcal{C}$-algebra $A$ is an object together with $\operatorname{Sym}_{j}$-equivariant maps

$$
\varphi(j) \otimes A^{j} \rightarrow A,
$$

thought of as actions, which are suitably associative and unital. Here $A^{j}$ represents $A^{\otimes j}$ and $A^{0}=\kappa$. An $A$-module $M$ is an object together with $\operatorname{Sym}_{j-1}$-equivariant maps

$$
\ell(j) \otimes A^{j-1} \otimes M \rightarrow M
$$

which are also suitably associative and unital. Note that we are taking our objects all from symmetric monoidal categories, so we do not need to distinguish where the operad lives from where the algebras live, but we have not eliminated the possibility of doing so. When we consider the entropy operad, the $n$-ary operations of the operad will be parametrized by rooted trees, while we will take algebras from the category of topological categories.

10.1. Operads and entropy. We first recall the recent construction of J. Baez, T. Fritz, and T. Leinster, [4], [5] of an operadic formalism for entropy, which is especially relevant to our setting and nicely displays the basic machinery.

Adopting the set theorists' convention, let us define natural numbers $n$ by $n=\{0, \ldots, n-1\}$. An ordered $n$-tuple will be denoted by $\left(a_{i}\right)_{i \in n}=\left(a_{0}, \ldots, a_{n-1}\right)$. Consider as our symmetric monoidal category the category of topological categories, denoted by Cat(Top), with $\otimes$ as the Cartesian product, and $\kappa$ as the one-point space. One can construct an operad, $\mathcal{P}$, out of probability distributions on finite sets. For each $j$, we define $\mathcal{P}(j)$ as the set of $j$-ary probability distributions, thought of as the $(j-1)$-simplex, $\Delta_{j-1} \subset \mathbb{R}^{j}$, and given the subspace topology. If $\left(p_{i}\right)_{i \in j} \in \mathcal{C}(j)$, and for $i \in\{1, \ldots, j\},\left(q_{i l}\right)_{l \in k_{i}} \in \mathcal{P}\left(k_{i}\right)$, we let

$\gamma\left(\left(p_{i}\right)_{i \in j} \otimes\left(q_{1 l}\right)_{l \in k_{0}} \otimes \cdots \otimes\left(q_{j l}\right)_{l \in k_{j-1}}\right)=\left(p_{i} q_{i l}\right)_{l \in k_{i}, i \in j} \in \mathcal{C}\left(k_{0}+\cdots+k_{j-1}\right)$.

Basically, this says that, given a binary variable $X \in\left(x_{i}\right)_{i \in j}$ with probability distribution $\left(p_{i}\right)_{i \in j}$, we refine the possible values of $X$, splitting up each $x_{i}$.

As a heuristic description of this procedure, imagine we are measuring physical systems and have suddenly discovered how to measure spin or some other quantity that we were ignorant of before. Now there are more distinguishable states that 
we can measure. We know the probability distribution of these new states given an old state $x_{i}$ : it is $\left(q_{i l}\right)_{l \in k_{i}}$, corresponding to new distinguishable states $\left(x_{i l}\right)_{l \in k_{i}}$. Now $X \in\left(x_{i l}\right)_{l \in k_{i}, i \in j}$ may take any of $k_{0}+\cdots+k_{j-1}$ values and must have the probability distribution $\left(p_{i} q_{i l}\right)_{l \in k_{i}, i \in j}$. We see that the unit in this operad is the unique probability distribution $(1) \in \mathcal{P}(1)$.

An important $\mathcal{P}$-algebra in Cat(Top) is given by the additive monoid $\mathbb{R}_{\geqslant 0}$. As a category, $\mathbb{R}_{\geqslant 0}$ is regarded as the one object category. the operad $\mathcal{P}$ acts trivially on objects since there is only one object. On maps, that is, on real numbers, we have

$$
\left(p_{i}\right)_{i \in j} \cdot\left(x_{i}\right)_{i \in j}=\sum_{i} p_{i} x_{i} .
$$

Since $\mathcal{P}$-algebras $A$ are also categories, we can define an internal $\mathcal{P}$-algebra in $A$ as a lax map $1 \rightarrow A$ of $\mathcal{P}$-algebras where 1 is the terminal $\mathcal{P}$-algebra in Cat (see [4], [5]) for details). This basically is an object $a \in A$ and, for each $p \in \mathcal{P}(j)$, a map $\alpha_{p}: p(a, \ldots, a) \rightarrow a$ such that

$$
\begin{aligned}
\alpha_{p \circ\left(q_{1}, \ldots, q_{n}\right)} & =\alpha_{p} \circ p\left(\alpha_{q_{1}}, \ldots, \alpha_{q_{n}}\right) \quad \text { for every } p \in \mathcal{P}(n) \text { and } q_{i} \in \mathcal{P}\left(m_{i}\right), \\
\alpha_{\sigma p} & =\alpha_{p} \quad \text { for every } p \in \mathcal{P}(n) \text { and } \sigma \in \operatorname{Sym}_{n}, \\
\alpha_{1} & =\eta
\end{aligned}
$$

For $\mathbb{R}_{\geqslant 0}$, there is only one object, so $a=\mathbb{R}_{\geqslant 0}$, and $\alpha$ is a map taking probability distributions to positive real numbers satisfying the following four axioms:

(1) for every $p \in \mathcal{P}(n)$ and $q_{i} \in \mathcal{P}\left(m_{i}\right)$,

$$
\alpha\left(p \circ\left(q_{1}, \ldots, q_{n}\right)\right)=\alpha(p)+\sum_{i} p_{i} \alpha\left(q_{i}\right) ;
$$

(2) $\alpha((1))=0$;

(3) for every $p \in \mathcal{P}(n)$ and $\sigma \in \operatorname{Sym}_{n}$,

$$
\alpha(\sigma p)=\alpha(p)
$$

(4) $\alpha: \mathcal{P}(n) \rightarrow \mathbb{R}_{\geqslant 0}$ is continuous for all $n$.

Note that, in the first of these, composition of maps in the one object category $\mathbb{R}_{\geqslant 0}$ is addition of real numbers. We require the last one since we are looking for functoriality in Cat(Top). As it turns out (see [4], [5]), by Faddeev's theorem [17], the only function satisfying these axioms, up to positive scalar multiples, is the Shannon entropy, Sh.

10.2. Binary guessing trees. Consider now a general binary information measure, $S:[0,1] \rightarrow \mathbb{R}_{\geqslant 0}$. We will assume that $S$ satisfies the identity axioms, so that we can keep our approach finite rather than full of infinite amounts of trivial flotsam. We can build an information measure on ternary variables in several ways. For example, if we are trying to guess at the value of $X$, which we know must be in $\left\{x_{1}, x_{2}, x_{3}\right\}$, using only yes-or-no questions, we could employ one of the following two strategies: 
(1) Is $X=x_{1}$ ? If not, is $X=x_{2}$ ?

(2) Is $X=x_{1}$ or $x_{2}$ ? If yes, is $X=x_{1}$ ?

Indeed, we see that any strategy that avoids asking trivial or irrelevant questions arises as one of these strategies with a permutation of $\{1,2,3\}$. This gives us $2 \cdot 3 !=12$ possible ternary information measures. There is a useful way of parametrizing these guessing strategies with rooted trees.

Proposition 10.1. Let $S$ be a binary information measure with identity. For each $n \geqslant 2$, there is a one-to-one correspondence between rooted full binary trees with $n$ leaves with labels in $\{1, \ldots, n\}$ and $n$-ary information measures arising from $S$.

Proof. Let $\boldsymbol{T}$ be a tree as above. We call such a tree an $(n, 2)$-tree. What it means to be full is that every vertex is either a leaf or has two children. We will see that eliminating the single-child nodes is equivalent to eliminating trivial and irrelevant questions from our set of possible questions, making it finite. To see what is the set of possible questions, consider that, if at a certain time we are certain that $X \in\left\{x_{1}, \ldots, x_{n}\right\}$, the yes-or-no questions available to us are exactly those of the form "is $X \in A$ ?", where $A$ is a subset of $\left\{x_{1}, \ldots, x_{n}\right\}$. We label the leaves of $\boldsymbol{T}$ with the possible values of $X$ according to their original labels $\left(i \mapsto x_{i}\right)$. The vertices which are not leaves are uniquely labeled with the list of $x_{i}$ which label the leaves of their subtree. The vertices will represent states of our knowledge of $X$ in that the labels will denote the possible values of $X$ given what we have already measured. Naturally, we begin at the root vertex, sure only that $X$ is one of the $x_{i}$. At any vertex which is not a leaf, there are two child subtrees: a left one, $\boldsymbol{L}$, and a right one, $\boldsymbol{D}$. Let $L$ be the set of leaf labels of $\boldsymbol{L}, D$ those of $\boldsymbol{D}$. The true value of $X$ must lie in either $L$ or $D$. Our question then is "is $X \in L$ ?". If the answer is yes, we move to the left child. If the answer is no, we move to the right child. At a leaf, we have ruled out all the possible values of $X$ except the one labeling our current vertex.

As an example, consider the rooted full binary tree in Figure 7:

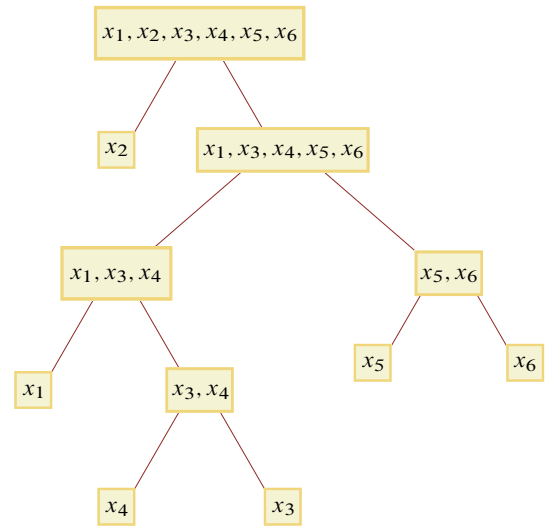

Figure 7. A rooted full binary tree. 
We see that $X$ lies in $\left\{x_{1}, \ldots, x_{6}\right\}$. Our strategy goes like this:

1. Our first question is "is $X=x_{2}$ ?".

1.1. If yes, we are done; $X=x_{2}$.

1.2. If no, we ask "is $X \in\left\{x_{1}, x_{3}, x_{4}\right\}$ ?".

1.2.1. If yes, we ask "is $X=x_{1}$ ?".

1.2.1.1. If yes, we are done; $X=x_{1}$.

1.2.1.2. If no, we ask "is $X=x_{4}$ ?".

1.2.1.2.1. If yes, we are done; $X=x_{4}$.

1.2.1.2.2. If no, we are also done; $X=x_{3}$.

1.2.2. If no, we ask "is $X=x_{5}$ ?".

1.2.2.1. If yes, we are done; $X=x_{5}$.

1.2.2.2. If no, we are also done; $X=x_{6}$.

Suppose these possible values occur with probabilities $p_{1}, \ldots, p_{6}$. We see that the information measure corresponding to the above tree is

$$
\begin{aligned}
S\left(p_{2}\right) & +\left(1-p_{2}\right) S\left(\frac{p_{1}+p_{4}+p_{3}}{1-p_{2}}\right)+\left(p_{1}+p_{4}+p_{3}\right) S\left(\frac{p_{1}}{p_{1}+p_{4}+p_{3}}\right) \\
& +\left(p_{4}+p_{3}\right) S\left(\frac{p_{4}}{p_{4}+p_{3}}\right)+\left(p_{5}+p_{6}\right) S\left(\frac{p_{5}}{p_{5}+p_{6}}\right) .
\end{aligned}
$$

Note that permuting the labels of the leaves permutes the $p_{i}$.

Conversely, since any question is of the form "is $X \in A$ ?" for some subsets $A$, we can build our tree inductively identifying $A$ with $L$ at a given vertex, and labeling with the possible values of $X$ as we go, beginning with the root. Any guessing strategy must exhaust the possibilities for $X$, so any tree constructed in this way will be a well-defined $(n, 2)$-tree. Clearly this is the inverse process to the one described above. As an example, suppose we want to guess at an $X \in\left\{x_{1}, x_{2}, x_{3}, x_{4}, x_{5}\right\}$. First we might ask if $X \in\left\{x_{1}, x_{2}, x_{4}\right\}$. If yes, we could ask if $X=x_{1}$. If not, if $X=x_{2}$. Backtracking, if $X \notin\left\{x_{1}, x_{2}, x_{4}\right\}$, we could ask whether $X=x_{5}$. This strategy exhausts the possibilities for $X$. It is represented by the tree in Figure 8: 


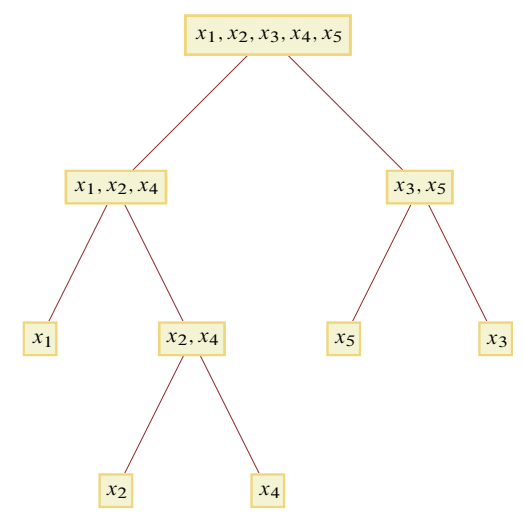

Figure 8. A guessing strategy.

Given an (n,2)-tree $\boldsymbol{T}$, there is a canonical way of arranging and parenthesizing an expression of the form $x_{1} \oplus_{S} \cdots \oplus_{S} x_{n}$ so that it may be evaluated. This is the same one given in the Catalan number identity [15]. We consider the tree $\boldsymbol{T}^{\prime}$ which is labeled $1, \ldots, n$ from left to right. Let $\sigma_{\boldsymbol{T}} \in \mathrm{Sym}_{n}$ be the permutation that sends the left-to-right labeling to the original one on $\boldsymbol{T}$. We define $\left(x_{1} \oplus S\right.$ $\left.\cdots \oplus_{S} x_{n}\right)_{\boldsymbol{T}}=\left(x_{\sigma_{\boldsymbol{T}}(1)} \oplus_{S} \cdots \oplus_{S} x_{\sigma_{\boldsymbol{T}}(n)}\right)_{\boldsymbol{T}^{\prime}}$. Thus, it suffices to consider the case when $\boldsymbol{T}$ is labeled left-to-right. In this case, there is a $1 \leqslant r<n$ such that for $1 \leqslant j \leqslant r, x_{j}$ is a label of a leaf of the left subtree $\boldsymbol{L}$, i.e., $x_{j} \in L$, and for all $r<j<n, x_{j} \in D$, where $\boldsymbol{D}$ is the right subtree of $\boldsymbol{T}$. Then we define inductively $\left(x_{1} \oplus_{S} \cdots \oplus_{S} x_{n}\right)_{T}=\left(x_{1} \oplus_{S} \cdots \oplus_{S} x_{r}\right)_{\boldsymbol{L}}+\left(x_{r+1} \oplus_{S} \cdots \oplus_{S} x_{n}\right)_{\boldsymbol{D}}$, with a tree with two children $\boldsymbol{T}_{2}$ giving $\left(x_{1} \oplus_{S} x_{2}\right) \boldsymbol{T}_{2}=x_{1} \oplus_{S} x_{2}$.

Theorem 10.2. Given an (n,2)-tree, $\boldsymbol{T}$, and a binary information measure $S$ with identity, the following holds:

$$
\left(x_{1} \oplus_{S} \cdots \oplus_{S} x_{n}\right)_{\boldsymbol{T}}=\min _{\sum p_{i}=1}\left(\sum p_{i} x_{i}-T S_{\boldsymbol{T}}\left(p_{1}, \ldots, p_{n}\right)\right) .
$$

Proof. Before we begin the proof in ernest, we illustrate the argument with an explicit example. We see the tree in Figure 7, which we denote $\boldsymbol{T}$, corresponds to the arrangement of parentheses $x_{1} \oplus_{S}\left(\left(x_{2} \oplus_{S}\left(x_{3} \oplus_{S} x_{4}\right)\right) \oplus_{S}\left(x_{5} \oplus_{S} x_{6}\right)\right)$ and the permutation $\sigma=(12)(34) \in \operatorname{Sym}_{6}$. We calculate

$$
\begin{aligned}
x_{1} \oplus_{S} & \left(\left(x_{2} \oplus_{S}\left(x_{3} \oplus_{S} x_{4}\right)\right) \oplus_{S}\left(x_{5} \oplus_{S} x_{6}\right)\right) \\
= & \min _{p_{1}}\left(p_{1} x_{1}+\left(1-p_{1}\right)\left(\left(x_{2} \oplus_{S}\left(x_{3} \oplus_{S} x_{4}\right)\right) \oplus_{S}\left(x_{5} \oplus_{S} x_{6}\right)\right)-T S\left(p_{1}\right)\right) \\
= & \min _{p_{1}}\left(p_{1} x_{1}+\left(1-p_{1}\right) \min _{p_{2}}\left(p_{2}\left(x_{2} \oplus_{S}\left(x_{3} \oplus_{S} x_{4}\right)\right)+\left(1-p_{2}\right)\left(x_{5} \oplus_{S} x_{6}\right)\right.\right. \\
& \left.\left.\quad-T S\left(p_{2}\right)\right)-T S\left(p_{1}\right)\right)
\end{aligned}
$$




$$
\begin{aligned}
= & \min _{p_{1}, p_{2}}\left(p_{1} x_{1}+\left(1-p_{1}\right) p_{2} \min _{p_{3}}\left(p_{3} x_{2}+\left(1-p_{3}\right)\left(x_{3} \oplus S x_{4}\right)-T S\left(p_{3}\right)\right)\right. \\
& +\left(1-p_{1}\right)\left(1-p_{2}\right) \min _{p_{4}}\left(p_{4} x_{5}+\left(1-p_{4}\right) x_{6}-T S\left(p_{4}\right)\right) \\
& \left.-T\left(S\left(p_{1}\right)+\left(1-p_{1}\right) S\left(p_{2}\right)\right)\right) \\
= & \min _{p_{1}, p_{2}, p_{3}, p_{4}, p_{5}}\left(p_{1} x_{1}+\left(1-p_{1}\right) p_{2} p_{3} x_{2}+\left(1-p_{1}\right) p_{2}\left(1-p_{3}\right) p_{5} x_{3}\right. \\
& +\left(1-p_{1}\right) p_{2}\left(1-p_{3}\right)\left(1-p_{5}\right) x_{4}+\left(1-p_{1}\right)\left(1-p_{2}\right) p_{4} x_{5} \\
& +\left(1-p_{1}\right)\left(1-p_{2}\right)\left(1-p_{4}\right) x_{6}-T\left(S\left(p_{1}\right)+\left(1-p_{1}\right) S\left(p_{2}\right)\right. \\
& +\left(1-p_{1}\right) p_{2} S\left(p_{3}\right)+\left(1-p_{1}\right)\left(1-p_{2}\right) S\left(p_{4}\right) \\
& \left.\left.+\left(1-p_{1}\right) p_{2}\left(1-p_{3}\right) S\left(p_{5}\right)\right)\right) .
\end{aligned}
$$

Now we make the substitution

$$
\begin{aligned}
& q_{1}=p_{1}, \\
& q_{2}=\left(1-p_{1}\right) p_{2} p_{3}, \\
& q_{3}=\left(1-p_{1}\right) p_{2}\left(1-p_{3}\right) p_{5}, \\
& q_{4}=\left(1-p_{1}\right) p_{2}\left(1-p_{3}\right)\left(1-p_{5}\right), \\
& q_{5}=\left(1-p_{1}\right)\left(1-p_{2}\right) p_{4}, \\
& q_{6}=\left(1-p_{1}\right)\left(1-p_{2}\right)\left(1-p_{4}\right) .
\end{aligned}
$$

We notice $q_{1}+\cdots+q_{6}=1$, and

$$
\begin{aligned}
& p_{1}=q_{1}, \\
& p_{2}=\left(q_{2}+q_{3}+q_{4}\right) /\left(1-q_{1}\right), \\
& p_{3}=q_{2} /\left(q_{2}+q_{3}+q_{4}\right), \\
& p_{4}=q_{5} /\left(q_{5}+q_{6}\right), \\
& p_{5}=q_{3} /\left(q_{3}+q_{4}\right) .
\end{aligned}
$$

Notice that these look like relative probabilities. This is no coincidence. Making this substitution above yields

$$
\begin{aligned}
x_{1} \oplus S & \left(\left(x_{2} \oplus S\left(x_{3} \oplus S x_{4}\right)\right) \oplus S\left(x_{5} \oplus S x_{6}\right)\right) \\
= & \min _{\sum q_{i}=1}\left(\sum q_{i} x_{i}-T\left(S\left(q_{1}\right)+\left(1-q_{1}\right) S\left(\frac{q_{2}+q_{3}+q_{4}}{1-q_{1}}\right)\right.\right. \\
& +\left(q_{2}+q_{3}+q_{4}\right) S\left(\frac{q_{2}}{q_{2}+q_{3}+q_{4}}\right)+\left(q_{3}+q_{4}\right) S\left(\frac{q_{3}}{q_{3}+q_{4}}\right) \\
& \left.+\left(q_{5}+q_{6}\right) S\left(\frac{q_{5}}{q_{5}+q_{6}}\right)\right) .
\end{aligned}
$$

Applying $\sigma$ we obtain

$\left(x_{1} \oplus_{S} x_{2} \oplus_{S} x_{3} \oplus_{S} x_{4} \oplus_{S} x_{5} \oplus_{S} x_{6}\right) \boldsymbol{T}=\min _{\sum p_{i}=1}\left(\sum p_{i} x_{i}-T S_{\boldsymbol{T}}\left(p_{1}, \ldots, p_{6}\right)\right)$, 
as the theorem claims.

Now we are ready to prove the theorem in general.

Lemma 10.3. Suppose that, at the root, the tree $\boldsymbol{T}$ has left subtree $\boldsymbol{L}$ with l leaves, and right subtree $\boldsymbol{D}$ with d leaves, and the leaves of $\boldsymbol{T}$ are labeled left to right. Then

$$
\begin{aligned}
& S_{\boldsymbol{T}}\left(p_{1}, \ldots, p_{l}, p_{l+1}, \ldots, p_{l+d}\right) \\
& =S\left(p_{1}+\cdots+p_{l}\right)+\left(p_{1}+\cdots+p_{l}\right) S_{\boldsymbol{L}}\left(\frac{p_{1}}{p_{1}+\cdots+p_{l}}, \ldots, \frac{p_{l}}{p_{1}+\cdots+p_{l}}\right) \\
& \quad+\left(p_{l+1}+\cdots+p_{l+d}\right) S_{\boldsymbol{D}}\left(\frac{p_{l+1}}{p_{l+1}+\cdots+p_{l+d}}, \ldots, \frac{p_{l+d}}{p_{l+1}+\cdots+p_{l+d}}\right) .
\end{aligned}
$$

Lemma 10.4. Suppose that, at the root, $\boldsymbol{T}$ has left subtree $\boldsymbol{L}$ with $l$ leaves and right subtree $\boldsymbol{D}$ with d leaves, and the leaves of $\boldsymbol{T}$ are labeled left to right. Then

$$
\begin{aligned}
& \left(x_{1} \oplus_{S} \cdots \oplus_{S} x_{l} \oplus_{S} x_{l+1} \oplus_{S} \cdots \oplus_{S} x_{l+d}\right)_{\boldsymbol{T}} \\
& \quad=\min _{p}\left(p\left(x_{1} \oplus_{S} \cdots \oplus_{S} x_{l}\right)_{\boldsymbol{L}}+(1-p)\left(x_{l+1} \oplus_{S} \cdots \oplus_{S} x_{l+d}\right)_{\boldsymbol{D}}-T S(p)\right) .
\end{aligned}
$$

The proof of both of these statements is immediate from the definitions.

Now, clearly the theorem holds when $\boldsymbol{T}$ has two leaves, and since our trees are full, we can use this as the base case in an induction.

Suppose the theorem holds for all trees with less than $n$ leaves. Let $\boldsymbol{T}$ be an $(n, 2)$-tree with leaves labeled from left to right. At the root, since $\boldsymbol{T}$ is full, $\boldsymbol{T}$ has nonempty left and right subtrees, $\boldsymbol{L}$ and $\boldsymbol{D}$, with $l>0$ and $d>0$ leaves, respectively, such that $l+d=n$, so $l, d<n$. By the inductive hypothesis and the second lemma above,

$$
\begin{aligned}
\left(x_{1} \oplus_{S} \cdots \oplus S x_{n}\right)_{T} \\
\quad=\min _{p}\left(p \min _{p_{1}+\cdots+p_{l}=1}\left(\sum p_{i} x_{i}-T S_{\boldsymbol{L}}\left(p_{1}, \ldots, p_{l}\right)\right)\right. \\
\left.\quad+(1-p) \min _{p_{l+1}+\cdots+p_{l+d}=1}\left(\sum p_{i} x_{i}-T S_{\boldsymbol{D}}\left(p_{l+1}, \ldots, p_{l+d}\right)\right)-T S(p)\right) .
\end{aligned}
$$

Make the substitution $q_{i}=p p_{i}$, for each $i \in\{1, \ldots, l\}$, and $q_{i}=(1-p) p_{i}$, for each $i \in\{l+1, \ldots, l+d\}$. Note that $q_{1}+\cdots+q_{l}=p$ and $q_{l+1}+\cdots+q_{l+d}=1-p$. This yields

$$
\begin{aligned}
\left(x_{1} \oplus_{S} \cdots \oplus_{S} x_{n}\right) \boldsymbol{T} \\
=\min _{\sum q_{i}=1}\left(\sum q_{i} x_{i}-T\left(\left(q_{1}+\cdots+q_{l}\right) S_{\boldsymbol{L}}\left(\frac{q_{1}}{q_{1}+\cdots+q_{l}}, \ldots, \frac{q_{l}}{q_{1}+\cdots+q_{l}}\right)\right.\right. \\
\quad+\left(q_{l+1}+\cdots+q_{l+d}\right) S_{\boldsymbol{D}}\left(\frac{q_{l+1}}{q_{l+1}+\cdots+q_{l+d}}, \ldots, \frac{q_{l+d}}{q_{l+1}+\cdots+q_{l+d}}\right) \\
\left.\left.\quad+S\left(q_{1}+\cdots+q_{l}\right)\right)\right),
\end{aligned}
$$


which by the first lemma is

$$
\left.\min _{\sum q_{i}=1}\left(\sum q_{i} x_{i}-T S_{T}\left(q_{1}, \ldots, q_{n}\right)\right)\right) .
$$

We need now show that this holds for arbitrary labelings of the leaves of $\boldsymbol{T}$. If $\sigma$ is a permutation of $\{1, \ldots, n\}$, then

$$
\begin{aligned}
\left(x_{\sigma(1)} \oplus_{S} \cdots \oplus_{S} x_{\sigma(n)}\right) \boldsymbol{T} & =\min _{\sum q_{i}=1}\left(\sum q_{i} x_{\sigma(i)}-T S_{\boldsymbol{T}}\left(q_{1}, \ldots q_{n}\right)\right) \\
& =\min _{\sum p_{i}=1}\left(\sum p_{i} x_{i}-T S_{\boldsymbol{T}}\left(p_{\sigma(1)}, \ldots, p_{\sigma(n)}\right)\right),
\end{aligned}
$$

where we have substituted $p_{i}=q_{\sigma^{-1}(i)}$. This proves the theorem.

The connection between these guessing games and the thermodynamics of mixing discussed in $\S 5$ can be intuited in the following way. The entropy of a system arises from considering the "correct counting" of states. In more words, some states are indistinguishable from others, and this affects their multiplicity in the partition sum. The entropy function tells us what the overall degree of distinguishability is. We can see this point of view in Boltzmann's famous equation asserting $S=k_{B} \log \Omega$, where $\Omega$ is the number of microstates which degenerate to a given macrostate. When we perform mixtures in a certain order, we are giving an order to this distinguishing process, as we are when we decide on an order to ask questions in a guessing game, distinguishing possible values from impossible values of the unknown variable.

10.3. General guessing trees. Now suppose that for each $n \in V \subseteq\{m \in \mathbb{N} \mid$ $m \geqslant 2\}$ we have an $n$-ary information measure $S_{n}$. We want to impose the following condition.

(1) (Coherence) Suppose that $n>m$ and, for all but $1<i_{1}<\cdots<i_{m}<n$, $p_{j}=0$. Then

$$
S_{n}\left(p_{1}, \ldots, p_{n}\right)=S_{m}\left(p_{i_{1}}, \ldots, p_{i_{m}}\right) .
$$

We can always write

$$
S_{n-1}\left(p_{1}, \ldots, p_{n-1}\right)=S_{n}\left(p_{1}, \ldots, p_{n-1}, 0\right),
$$

so that we can take $V$ as an initial segment of $\mathbb{N}_{\geqslant 2}$. This way, we can instead think about $v=\sup V$. Many definitions of entropies have $v=\infty$. Examples include the Shannon, Renyi, and Tsallis entropies. These are generally defined by functions $f$, $g$ such that

$$
S_{n}\left(p_{1}, \ldots, p_{n}\right)=f\left(\sum_{1 \leqslant i \leqslant n} g\left(p_{i}\right)\right) .
$$

Any entropy of this form trivially satisfies the coherence axiom.

In this more general setting, we can ask any question with up to $v$ possible answers. This potentially gives us many new ways to play guessing games, or equivalently, 
to build more general information measures. For example, if $v \geqslant 5$, then we can measure a 12-ary random variable $X \in\left\{x_{1}, \ldots, x_{12}\right\}$ by asking first whether $X \in$ $\left\{x_{1}, \ldots, x_{5}\right\}$. If yes, we simply measure the value of $X$. Otherwise, ask which of $\left\{x_{6}, x_{7}, x_{8}\right\},\left\{x_{9}, x_{12}\right\}$, or $\left\{x_{10}, x_{11}\right\}$ contains $X$, and then simply measure the value of $X$ (note that order may matter: $S_{n}$ may not be symmetric). This gives us information

$$
\begin{aligned}
& S_{2}\left(p_{1}+\cdots+p_{5}, p_{6}+\cdots+p_{12}\right) \\
& \quad+\left(p_{1}+\cdots+p_{5}\right) S_{5}\left(\frac{p_{1}}{p_{1}+\cdots+p_{5}}, \ldots, \frac{p_{5}}{p_{1}+\cdots+p_{5}}\right) \\
& \quad+\left(p_{6}+\cdots+p_{12}\right) S_{3}\left(\frac{p_{6}+p_{7}+p_{8}}{p_{6}+\cdots+p_{12}}, \frac{p_{9}+p_{12}}{p_{6}+\cdots+p_{12}}, \frac{p_{10}+p_{11}}{p_{6}+\cdots+p_{12}}\right) \\
& \quad+\left(p_{6}+p_{7}+p_{8}\right) S_{3}\left(\frac{p_{6}}{p_{6}+p_{7}+p_{8}}, \frac{p_{7}}{p_{6}+p_{7}+p_{8}}, \frac{p_{8}}{p_{6}+p_{7}+p_{8}}\right) \\
& \quad+\left(p_{9}+p_{12}\right) S_{2}\left(\frac{p_{9}}{p_{9}+p_{12}}, \frac{p_{12}}{p_{9}+p_{12}}\right) \\
& \quad+\left(p_{10}+p_{11}\right) S_{2}\left(\frac{p_{10}}{p_{10}+p_{11}}, \frac{p_{11}}{p_{10}+p_{11}}\right),
\end{aligned}
$$

where now we write $S_{2}$ as a two-variable function for consistency. We see something extremely similar to the binary case is happening here.

Proposition 10.5. Let $n, v \geqslant 2$, and suppose that for each $2 \leqslant j<v+1$ we have a $j$-ary information measure $S_{j}$, and these together satisfy the coherence axiom. Guessing strategies of $n$-ary random variables where we allow questions of up to $v$ possible answers are in bijective correspondence with the set of $(n, v)$-trees, rooted trees with labeled leaves such that every vertex is either a leaf or has between 2 and $v$ children.

Proof. Every relevant question that can be asked is of the form "which of $A_{1}, \ldots, A_{m}$ contains $X$ ?" for certain disjoint subsets $A_{1}, \ldots, A_{m}$. We identify these subsets with the leaves of the $m$ subtrees extending from the current vertex, once again identifying the vertices with states of our knowledge of $X$.

For example, from the previous algorithm we have the tree in Figure 9.

Conversely, to go from an $(n, v)$-tree to a guessing strategy one must only follow the tree to its leaves.

Now we have some basic $n$-ary functions for more than just $n=2$. Namely, we can define

$$
x_{1} \oplus_{S} \cdots \oplus S x_{n}:=\min _{\sum p_{i}=1}\left(\sum_{1 \leqslant i \leqslant n} p_{i} x_{i}-T S_{n}\left(p_{1}, \ldots, p_{n}\right)\right) .
$$

This has a thermodynamic interpretation of a simultaneous mixing of $n$ gas species. We have the following result. 


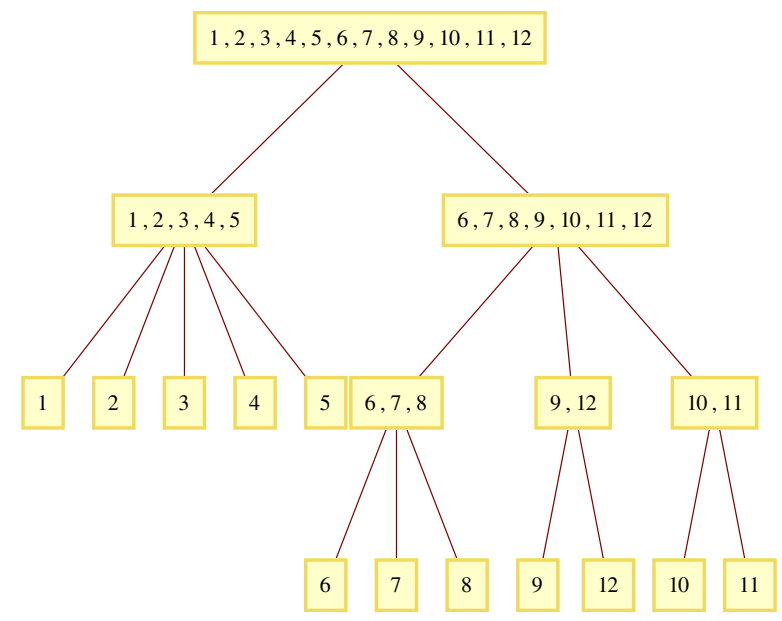

Figure 9. An $(n, v)$-tree.

Proposition 10.6. Let $n>2$. The following hold.

(1) For every $j$,

$$
\begin{aligned}
x_{1} \oplus_{S} & \cdots \oplus_{S} x_{j} \oplus_{S} \infty \oplus_{S} x_{j+2} \oplus_{S} \cdots \oplus_{S} x_{n} \\
& =x_{1} \oplus_{S} \cdots \oplus_{S} x_{j} \oplus_{S} x_{j+2} \oplus_{S} \cdots \oplus_{S} x_{n}
\end{aligned}
$$

if and only if the $S_{n}$ share the coherence property. For $n=2$ this is the identity property.

(2) $x_{1} \oplus_{S} \cdots \oplus_{S} x_{n}$ is symmetric if and only if $S_{n}$ is symmetric. For $n=2$ this is commutativity.

The proof of this fact is immediate.

We can generalize the parentheses correspondence to $(n, v)$-trees: to any $(n, v)$ tree $\boldsymbol{T}$ we can associate a unique $n$-ary function $\left(x_{1} \oplus_{S} \cdots \oplus_{S} x_{n}\right)_{T}$ given by arranging parentheses according to $\boldsymbol{T}$ and $x_{i}$ according to the labels on the leaves.

For example, to the tree above we associate

$\left(x_{1} \oplus_{S} x_{2} \oplus_{S} x_{3} \oplus_{S} x_{4} \oplus_{S} x_{5}\right) \oplus_{S}\left(\left(x_{6} \oplus_{S} x_{7} \oplus_{S} x_{8}\right) \oplus_{S}\left(x_{9} \oplus_{S} x_{12}\right) \oplus_{S}\left(x_{10} \oplus_{S} x_{11}\right)\right)$

We have then the analog for $(n, v)$-trees of Lemmas 10.3 and 10.4.

Lemma 10.7. Suppose that the root of an $(n, v)$-tree $\boldsymbol{T}$ has sub- $\left(l_{j}, v\right)$-trees (resp. from left to right) $\boldsymbol{A}_{1}, \ldots, \boldsymbol{A}_{m}$, and the leaves of $\boldsymbol{T}$ are labeled left to right. Let 
$L_{j}=l_{1}+\cdots+l_{j}$ and $L_{0}=0$. Then the following holds.

$$
\begin{aligned}
S_{\boldsymbol{T}}\left(p_{1}, \ldots, p_{n}\right)= & \sum_{1 \leqslant j \leqslant m}\left(p_{L_{j-1}+1}+\cdots+p_{L_{j}}\right) \\
& S_{\boldsymbol{A}_{j}}\left(\frac{p_{L_{j-1}+1}}{p_{L_{j-1}+1}+\cdots+p_{L_{j}}}, \ldots, \frac{p_{L_{j}}}{p_{L_{j-1}+1}+\cdots+p_{L_{j}}}\right) \\
& +S_{m}\left(p_{L_{0}+1}+\cdots+p_{L_{1}}, \ldots, p_{L_{m-1}+1}+\cdots+p_{L_{m}}\right)
\end{aligned}
$$

Lemma 10.8. Suppose the root of an $(n, v)$-tree $\boldsymbol{T}$ has sub- $\left(l_{j}, v\right)$-trees (resp. from left to right) $\boldsymbol{A}_{1}, \ldots, \boldsymbol{A}_{m}$, and the leaves of $\boldsymbol{T}$ are labeled left to right. Let $L_{j}=$ $l_{1}+\cdots+l_{j}, L_{0}=0$. Then the following holds:

$$
\begin{aligned}
\left(x_{1} \oplus_{S} \cdots \oplus_{S} x_{n}\right)_{\boldsymbol{T}}= & \min _{\sum q_{i}=1}\left(q_{1}\left(x_{1} \oplus_{S} \cdots \oplus_{S} x_{l_{1}}\right)+\cdots\right. \\
& \cdots+q_{m}\left(x_{l_{1}+\cdots+l_{m-1}+1} \oplus_{S} \cdots \oplus_{S} x_{l_{1}+\cdots+l_{m}}\right) \\
& \left.-T S_{m}\left(q_{1}, \ldots, q_{m}\right)\right) .
\end{aligned}
$$

As before, both of these are immediate from the definitions. Finally, we have the theorem:

Theorem 10.9. Given an $(n, v)$-tree $\boldsymbol{T}$, and for each $2 \leqslant j \leqslant n$ an information measure $S_{j}$ such that together they satisfy the coherence axioms, the following holds:

$$
\left(x_{1} \oplus_{S} \cdots \oplus_{S} x_{n}\right)_{T}=\min _{\sum p_{i}=1}\left(\sum p_{i} x_{i}-T S_{\boldsymbol{T}}\left(p_{1}, \ldots, p_{n}\right)\right) .
$$

Proof. Once again we proceed by strong induction on the number of leaves. We know the theorem holds for $n=2$. Suppose the theorem holds for every $(m, v)$-tree with $m<n$. Let $\boldsymbol{T}$ be an $(n, v)$-tree with leaves labeled from left to right. $\boldsymbol{T}$ has $k \geqslant 2$ sub- $\left(l_{i}, v\right)$-trees starting at the root (resp. from left to right) $\boldsymbol{A}_{1}, \ldots, \boldsymbol{A}_{k}$ with $l_{i}>0$. We must have $l_{1}+\cdots+l_{k}=n$, so $l_{i}<n$. By the inductive hypothesis and the second lemma above, we obtain

$$
\begin{aligned}
\left(x_{1} \oplus S\right. & \left.\cdots \oplus S x_{n}\right) \boldsymbol{T} \\
= & \min _{\sum q_{i}=1}\left(q _ { 1 } \operatorname { m i n } _ { p _ { 1 } + \cdots + p _ { l _ { 1 } } = 1 } \left(p_{1} x_{1}+\cdots+p_{l_{1}} x_{l_{1}}-T S_{\boldsymbol{A}_{1}}\left(p_{1}, \ldots, p_{l_{1}}\right)+\cdots\right.\right. \\
& \cdots+q_{k} \min _{p_{l_{1}+\cdots+l_{k-1}+1}+\cdots+p_{l_{1}+\cdots+l_{k}}=1}\left(\sum_{j=l_{1}+\cdots+l_{k-1}+1}^{l_{1}+\cdots+l_{k}} p_{j} x_{j}\right. \\
& \left.-T S_{A_{k}}\left(p_{l_{1}+\cdots+l_{k-1}+1}, \ldots, p_{l_{1}+\cdots+l_{k}}\right)\right) \\
& \left.-T S_{k}\left(p_{1}+\cdots+p_{l_{1}}, \ldots, p_{l_{1}+\cdots+l_{k-1}+1}+\cdots+p_{n}\right)\right) .
\end{aligned}
$$

For each $i \in\{1, \ldots, k\}$, and each $j \in\left\{l_{1}+\cdots+l_{i-1}+1, \ldots, l_{1}+\cdots+\right.$ $\left.l_{i}\right\}$, where we define $l_{0}=0$, we make the substitution $\tilde{q}_{j}=q_{i} p_{j}$. That way, 
$\sum_{j=l_{1}+\cdots+l_{i-1}}^{l_{1}+\cdots+l_{i}}+\tilde{q}_{j}=q_{i}$, so we have

$$
\begin{aligned}
\left(x_{1} \oplus S\right. & \left.\cdots \oplus S x_{n}\right) \boldsymbol{T} \\
= & \min _{\sum \tilde{q}_{j}=1}\left(\sum \tilde{q}_{j} x_{j}-T\left(\left(\tilde{q}_{1}+\cdots+\tilde{q}_{l_{1}}\right) S_{\boldsymbol{A}_{1}}\left(\frac{\tilde{q}_{1}}{\tilde{q}_{1}+\cdots+\tilde{q}_{l_{1}}}, \ldots\right)+\cdots\right.\right. \\
& \left.\quad+\left(\tilde{q}_{l_{1}+\cdots+l_{k-1}+1}+\cdots+\tilde{q}_{n}\right) S_{\boldsymbol{A}_{k}}\left(\frac{\tilde{q}_{l_{1}+\cdots+l_{k-1}+1}}{\tilde{q}_{l_{1}+\cdots+l_{k-1}+1}+\cdots+\tilde{q}_{n}}, \ldots\right)\right) .
\end{aligned}
$$

By Lemma 10.7, this equals

$$
\min _{\sum \tilde{q}_{j}=1}\left(\sum \tilde{q}_{j} x_{j}-T S_{\boldsymbol{T}}\left(\tilde{q}_{1}, \ldots, \tilde{q}_{n}\right)\right)
$$

Now let $\sigma$ be any permutation of $\{1, \ldots, n\}$. We see that

$$
\begin{aligned}
\left(x_{\sigma(1)} \oplus_{S} \cdots \oplus S x_{\sigma(n)}\right) \boldsymbol{T} & =\min _{\sum q_{i}=1}\left(\sum q_{i} x_{\sigma(i)}-T S_{\boldsymbol{T}}\left(q_{1}, \ldots q_{n}\right)\right) \\
& =\min _{\sum p_{i}=1}\left(\sum p_{i} x_{i}-T S_{\boldsymbol{T}}\left(p_{\sigma(1)}, \ldots, p_{\sigma(n)}\right)\right),
\end{aligned}
$$

where we have substituted $p_{i}=q_{\sigma^{-1}(i)}$. This proves the theorem.

10.4. Information algebra. We define $\mathcal{T}_{v}(n)$ to be the class of $(n, v)$-trees such that $\mathcal{T}_{v}(0)$ contains only the empty graph and $\mathcal{T}_{v}(1)$ contains only the unique oneleavèd $(n, v)$-tree. We put an operad structure on the union of these collections, $\mathcal{T}$ with composition given by leaf-to-root composition of trees, which is clearly unital, associative, and Sym-equivariant. Our underlying category is the cartesian monoidal category of sets of graphs, with $\kappa=\mathcal{T}_{v}(1)$. Note that this unital operad structure, if also given a free group structure, forms the well-known $A_{\infty}$-operad.

Consider the one-object topological category, $R$, and a coherent set

$$
\left\{S_{j}: I^{j} \rightarrow \mathbb{R}_{\geqslant 0} \mid 2 \leqslant j<v+1\right\}
$$

of information measures. For each $n \geqslant 2$, and each $\boldsymbol{T} \in \mathcal{T}(n)$, we define

$$
\boldsymbol{T}\left(x_{1}, \ldots, x_{n}\right)=\min _{\sum p_{i}=1}\left(\sum p_{i} x_{i}-T S_{T}\left(p_{1}, \ldots, p_{n}\right)\right) .
$$

With the definition of $S_{\boldsymbol{T}}$, as in the previous section. For $\mathcal{T}(1)$, we define $(x)_{\boldsymbol{T}}=x$, for $\mathcal{T}_{v}(0)=\kappa$, we define ()$_{T}=\infty$.

By Theorem 10.9 above, this is the same as $\left(x_{1} \oplus_{S} \cdots \oplus_{S} x_{n}\right)_{T}$, which clearly behaves well under composition of trees and the action of $\operatorname{Sym}_{n}$, so this makes $R$ a $\mathcal{T}$-algebra, which we call the information algebra $(R, S)$. This characterizes the complete algebraic structure of the Witt semiring $R$ over $K$ arising from $S$. The next proposition is written in the original convention for semifields and summarizes some characteristics of this action, each of which are immediate from the definitions. 
Proposition 10.10. Let $\boldsymbol{T} \in \mathcal{T}(n), x_{1}, \ldots, x_{n}, y \in K, \alpha \in \mathbb{R} \geqslant 0$. Then the following hold.

(1) The $\mathcal{T}$-algebra structure on $R$ is additive: for all $1 \leqslant j \leqslant n$,

$$
\begin{aligned}
& \boldsymbol{T}\left(x_{1}, \ldots, x_{j-1}, x_{j}+y, x_{j+1}, \ldots, x_{n}\right) \\
& \quad=\boldsymbol{T}\left(x_{1}, \ldots, x_{j}, \ldots, x_{n}\right)+\boldsymbol{T}\left(x_{1}, \ldots, y, \ldots, x_{n}\right) .
\end{aligned}
$$

(2) Multiplication distributes over the $\mathcal{T}$-algebra structure:

$$
y\left(x_{1} \oplus_{S} \cdots \oplus_{S} x_{n}\right)_{T}=\left(y x_{1} \oplus_{S} \cdots \oplus_{S} y x_{n}\right)_{T} .
$$

(3) The $\mathcal{T}$-algebra structure also satisfies

$$
\left(x_{1} \oplus_{S} \cdots \oplus_{S} x_{n}\right)_{T}^{\alpha}(T)=\left(x_{1}^{\alpha} \oplus_{S} \cdots \oplus_{S} x_{n}^{\alpha}\right)_{T}(\alpha T) .
$$

The relations which are most natural to consider are of the form

$$
\boldsymbol{T}_{1}\left(x_{1}, \ldots, x_{n}\right)=\boldsymbol{T}_{2}\left(x_{1}, \ldots, x_{n}\right) \quad \text { for all } x_{i} \in R,
$$

where $\boldsymbol{T}_{1}$ and $\boldsymbol{T}_{2}$ are $(n, v)$-trees acting on the information algebra $(R, S)$. The reason is that we can interpret this as an equivalence of guessing strategies, so these are exactly the kind of relations that would define an information measure. Note it is not just $\boldsymbol{T}_{1}$ and $\boldsymbol{T}_{2}$ which are affected by this relation. Because composition of trees gives the composition of their actions on $R$, whenever some tree can be written $\boldsymbol{A} \circ \boldsymbol{T}_{1} \circ\left(\boldsymbol{A}_{1}, \ldots, \boldsymbol{A}_{n}\right)$, this is equivalent to $\boldsymbol{A} \circ \boldsymbol{T}_{2} \circ\left(\boldsymbol{A}_{1}, \ldots, \boldsymbol{A}_{n}\right)$. The equivalence classes of these trees for some fixed set of relations $\mathbf{R}$, defines a quotient operad $\mathcal{T} / \mathbf{R}$ which is the set of possible guessing strategies up to equivalence under the information measure. The terminal object in this construction is the one with exactly one $(m, v)$-tree for each $m$, which is precisely the quotient operad arising from the Shannon entropy.

However, one quickly finds that these simple relations are inadequate for describing the full range of information measures. If we have an equivalence of trees, we can always prune corresponding leaves by inserting the identity, $\infty$ in the current notation, in the place of that variable. For binary information measures, one has the following fact.

Proposition 10.11. Suppose that $S$ is a commutative binary information measure, and $\boldsymbol{T}_{1}, \boldsymbol{T}_{2}$ are $(n, 2)$-trees. Either $\boldsymbol{T}_{1}=\boldsymbol{T}_{2}$ is implied by commutativity or implies associativity, hence forces $S$ to be the Shannon entropy.

Proof. We proceed by induction on $n$. When $n=3$, one checks the above is true by simply checking each case. Suppose the theorem holds for all $m<n$. By pruning a leaf, we see that either the relation implies associativity or the pruned subtrees are equal. In the case of the latter, prune a different leaf and we see the theorem holds. 
Thus, one may wish to pass into a setting where we may consider linear combinations of trees, i.e., we can put a free vector space structure on our original operad. This gives us an $A_{\infty}$ operad, with the action on the information algebra $R$ extending uniquely under the Frobenius action.

Let us now consider what an internal $\mathcal{T}$-algebra in $R$ is. For each $n$, this is a continuous map $\alpha_{n}: \mathcal{T}(n) \rightarrow R$ such that the following hold:

(1) for all $\boldsymbol{T} \in \mathcal{T}(n)$ and $\boldsymbol{A}_{1} \in \mathcal{T}\left(m_{1}\right), \ldots, \boldsymbol{A}_{n} \in \mathcal{T}\left(m_{n}\right)$,

$$
\alpha_{m_{1}+\cdots+m_{n}} \boldsymbol{T} \circ\left(\boldsymbol{A}_{1}, \ldots, \boldsymbol{A}_{n}\right)=\alpha_{n}(\boldsymbol{T})+\boldsymbol{T}\left(\alpha_{m_{1}}\left(\boldsymbol{A}_{1}\right), \ldots, \alpha_{m_{n}}\left(\boldsymbol{A}_{n}\right)\right) ;
$$

(2) for all $\boldsymbol{T} \in \mathcal{T}(n)$ and $\sigma \in \operatorname{Sym}_{n}$,

$$
\alpha_{n}(\sigma \boldsymbol{T})=\alpha_{n}(\boldsymbol{T})
$$

(3) the following condition also holds:

$$
\alpha_{1}(\mathcal{T}(1))=0 .
$$

To simplify notation, we will suppress the subscripts on $\alpha$ and just consider $\alpha: \mathcal{T} \rightarrow \mathbb{R}_{\geqslant 0}$. The second condition above just says that $\alpha(\boldsymbol{T})$ does not depend on the labels of $\boldsymbol{T}$.

For each $2 \leqslant n<v+1$ we define $h_{n} \in \mathbb{R}_{\geqslant 0}$ as the unique value $\alpha$ takes on the $(n, v)$-trees with $n+1$ vertices, that is, those corresponding to $S_{n}$.

Every tree in $\mathcal{T}$ is built from these basic trees, and by the first condition above, so is $\alpha(\boldsymbol{T})$.

If at the root $\boldsymbol{T}$ has subtrees $\boldsymbol{A}_{1}, \ldots, \boldsymbol{A}_{n}$ from left to right, then

$$
\alpha(\boldsymbol{T})=h_{n}+\alpha\left(\boldsymbol{A}_{1}\right) \oplus_{S} \cdots \oplus_{S} \alpha\left(\boldsymbol{A}_{n}\right) .
$$

For the tree in Figure 9, this gives

$$
h_{2}+h_{5} \oplus_{S}\left(h_{3}+h_{3} \oplus_{S} h_{2} \oplus_{S} h_{2}\right) .
$$

We see that + goes down the tree, and $\oplus_{S}$ goes across the tree.

It is easy to see that $h_{3} \geqslant h_{3} \oplus_{S} h_{2} \oplus_{S} h_{2}$, and $h_{2} \geqslant h_{5} \oplus_{S}\left(h_{3} \oplus_{S} h_{2} \oplus_{S} h_{2}\right)$, so this can be simplified to

$$
h_{5} \oplus_{S}\left(h_{3} \oplus_{S} h_{2} \oplus_{S} h_{2}\right),
$$

which we see can be obtained through a different recursion strategy. Instead of picking off the subtrees at the root, we could pick off the basic subtrees just above the leaves. This is just another way of writing $\boldsymbol{T}$ as a composition of trees, and puts the recursion into the second term rather than the first in (10.1).

Because of this recursion, every internal $\mathcal{T}$-algebra of $\mathbb{R}_{\geqslant 0}$ is determined by the sequence $\left(h_{j}\right)_{2 \leqslant j<v+1}$ (by the third condition above, implicity $h_{1}=0$ ).

When $R=\mathbb{R}_{\geqslant 0}^{\max , *}$, and we use $S=\mathrm{Sh}$, the Shannon entropy, then $x \oplus_{S} y=$ $\left(x^{1 / T}+y^{1 / T}\right)^{T}$, so the above becomes

$$
\alpha(\boldsymbol{T})=\max \left(h_{n},\left(\alpha\left(\boldsymbol{A}_{1}\right)^{1 / T}+\cdots+\alpha\left(\boldsymbol{A}_{n}\right)^{1 / T}\right)^{T}\right) .
$$




\section{Further perspectives and directions}

We sketch here some possible further directions where the notion of thermodynamic semirings may prove useful.

11.1. Information geometry. Information geometry was developed [2], [3], [22] as a way to encode, using methods based on Riemannian geometry, statistical information, such as how to infer unobserved variables on the basis of observed ones by reducing conditional joint probabilities to marginal distributions.

We consider a smooth univariate binary statistical $n$-manifold $\mathcal{Q}$ as in Definition 8.1 parameterized by $\eta \in X \subset \mathbb{R}^{n}$. One may deal with the multivariate case similarly.

The Fisher information metric (see [3]) on information manifolds is given by

$$
g_{i j}(\theta)=\int \frac{\partial \ln p(x ; \theta)}{\partial \theta_{i}} \frac{\partial \ln p(x ; \theta)}{\partial \theta_{j}} d x,
$$

and it defines a Riemannian metric on a statistical manifold $\mathcal{Q}$.

Another important notion in information geometry is that of e-flat and $m$-flat submanifolds, which we recall here.

A submanifold $\delta \subset Q$ is e-flat if, for all $t \in[0,1]$ and all $p(\eta)$ and $q(\eta)$ in $\delta$, the mixture $\log r(\eta, t)=t \log p(\eta)+(1-t) \log q(\eta)+c(t)$, with $c(t)$ a normalization factor, is also in $\delta$.

A submanifold $\delta \subset Q \mathcal{Q}$ is m-flat if, for all $t \in[0,1]$ and all $p(\eta)$ and $q(\eta)$ in $\delta$, the mixture $r(\eta, t)=t p(\eta)+(1-t) q(\eta)$ is also in $\delta$.

One-dimensional e-flat or m-flat manifolds are called e-geodesics and m-geodesics, respectively. In information geometry, maximum posterior marginal optimization is achieved by finding the point on an e-flat submanifold $S$ that minimizes the KL divergence, see [3], [22]. It turns out that the point on an e-flat submanifold $\delta$ that minimizes the KL divergence also minimizes the Riemannian metric given by the Fisher information metric.

More precisely, when considering the $\mathrm{KL}$ divergences $\operatorname{KL}(p ; q(\eta))$, where $q(\eta)$ varies in an e-flat submanifold $\delta$ of the given information manifold $\mathcal{Q}$, there is a unique point $q(\eta)$ in $\delta$ that minimizes $\operatorname{KL}(p ; q(\eta))$ and it is given by the point where the m-geodesic from $p$ meets $S$ orthogonally with respect to the Fisher information metric (see Theorem 1 of [22]).

Thus, from the point of view of information geometry, it seems especially interesting to look at cases of the thermodynamic semiring structures

$$
x(\eta) \oplus_{\mathrm{KL}_{q(\eta)}} y(\eta)=\sum_{p} \rho^{-\mathrm{KL}(p ; q(\eta))} x(\eta)^{p} y(\eta)^{1-p}
$$

for distributions $q(\eta)$ that vary along e-flat submanifolds of information manifolds and recast some Riemannian aspects of information geometry in terms of algebraic properties of the thermodynamic semirings. 
11.2. Tropical geometry. Most of our results have a very natural thermodynamic interpretation when written explicitly in the case of the tropical semifield (seen as a prototype example of characteristic one semiring as in [10], [11]). Thus, besides the original motivation arising in the context of $\mathbb{F}_{1}$ geometry, it is possible that the theory of thermodynamic semirings we developed here may have some interesting applications in the setting of tropical geometry [24].

The use of tropical geometry in the context of probabilistic inference in statistical models was recently advocated in [41]. In that approach one considers polynomial maps from a space of parameters to the space of joint probability distributions on a set of random variables. These give statistical models described by algebraic varieties. The tropicalization of the resulting algebraic variety is then used as a model for parametric inference, for instance, by interpreting marginal probabilities as coordinates of points on the variety.

It would therefore seem interesting to extend the encoding of thermodynamic and information-theoretic properties into the additive structure of the semiring to the broader context of tropical varieties. In particular one can consider the patchworking process, where operations are performed on the "quantized" varieties, and then the limit in the Maslov dequantization, corresponding to the residue morphism $T \rightarrow 0$, is performed, obtaining the new tropical variety.

Observe, for instance, that in the usual setting of tropical geometry, in passing from an algebraic variety to its tropicalization, starting with a polynomial $f$ defining a hypersurface $V$ in $\left(\mathbb{C}^{*}\right)^{n}$, one can proceed by first considering an associated Maslov dequantization, given by a one-parameter family $f_{h}$, whose zero set one denotes by $V_{h}$. One then considers the amoeba obtained by mapping $V_{h}$ to $\mathbb{R}^{n}$ under the map $\log _{h}\left(z_{1}, \ldots, z_{n}\right)=\left(h \log \left|z_{1}\right|, \ldots, h \log \left|z_{n}\right|\right)$. One obtains in this way the amoeba $\mathcal{A}_{h}=\log _{h}\left(V_{h}\right)$. As we send the parameter $h \rightarrow 0$, the subsets $\mathcal{A}_{h} \subset \mathbb{R}^{n}$ converge in the Hausdorff metric to the tropical variety $\operatorname{Tro}(V)$, see [30]. For example, for a polynomial of the form $f(x)=\sum_{k} a_{k} x^{k}$, one obtains $f_{h}(x)$ by passing to $a_{k}=e^{b_{k}}$ and $x^{k}=e^{k t}$, so that one can then replace $v=\log \left(\sum_{k} e^{k t+b_{k}}\right)$ by the deformed $v_{h}=h \log \left(\sum_{k} e^{\left(k t+b_{k}\right) / h}\right)$, which in turn defines the dequantized family $f_{h}(x)$.

By comparing with Proposition 4.3, one can see that the Maslov dequantization can be expressed in terms of the operation $\oplus_{\mathrm{Sh}, T}$, where the dequantization parameter $h$ plays the role of the temperature $T$, as also observed in [10]. Therefore, one can introduce variants of the Maslov dequantization procedure, based on other operations $\oplus_{S, T}$, for other choice of information measures. In particular, one can consider dequantizations based on various $n$-ary information measures of the form

$$
\left(x_{1} \oplus_{S, T} \cdots \oplus_{S, T} x_{n}\right)_{T}=\min _{\sum_{i} p_{i}=1}\left(\sum_{i} p_{i} x_{i}-T S_{T}\left(p_{1}, \ldots, p_{n}\right)\right),
$$

with the data labeled by trees $\boldsymbol{T}$, as we described in $§ 10$ above.

While one can expect that the tropical limit itself will be independent of the use of different information measures in the dequantization procedure, operations performed at the level of the amoebas $\mathcal{A}_{h}$ will likely have variants with different properties when 
the Shannon entropy is replaced by other information measures of the kind considered in this paper.

11.3. The thermodynamics of $\mathbb{R}^{\mathrm{un}}$. In the characteristic $p$ case, the functoriality of the Witt construction provides a way to construct extensions of the field of $p$-adic numbers $\mathbb{Q}_{p}=\operatorname{Frac}\left(\mathbb{Z}_{p}\right)$ using the fact that $\mathbb{Z}_{p}=\mathbb{W}_{p}\left(\mathbb{F}_{p}\right)$, and applying the same Witt functor to extensions $\mathbb{F}_{q}$. This gives $\mathbb{W}_{p}\left(\mathbb{F}_{q}\right)=\mathbb{Z}_{p}\left[\zeta_{q-1}\right]$, which is the valuation ring of an unramified extension $\mathbb{Q}_{p}\left(\zeta_{q-1}\right)$ of $\mathbb{Q}_{p}$, see [33].

It was observed in $\S 7$ of [10] that, in the case of the characteristic one version of the Witt construction, when one considers the $\oplus_{\mathrm{Sh}, T}$ simultaneously for all possible temperatures $T$, one can describe a candidate analog of "unramified extension" $\mathbb{R}^{\text {un }}$ in terms of analogs of Teichmüller characters given in the form $\chi_{T}(f)=f(T)^{1 / T}$ and an analog of the residue morphism of the form $\epsilon(f)=\lim _{T \rightarrow 0} \chi_{T}(f)(T)^{T}$.

We can formulate this in the general case. We find, first of all that the Frobenius lifts do not depend on the information measure.

Proposition 11.1. If $R$ is a thermodynamic semiring over a suitably nice semifield $K$ defined by the information measure $S$, then the Frobenius lifts from $K$ to $R$ in such a way that

$$
F_{r}(x(T))=x(T / r)^{r} .
$$

Proof. We see that this is a result of the general form of the temperature dependence in the current context. In symbols, we are looking for

$$
\begin{aligned}
F_{r}\left(x(T) \oplus_{S} y(T)\right) & =\sum e^{r f(T) S(\alpha)} x(f(T))^{r \alpha} y(f(T))^{r(1-\alpha)} \\
& =\sum e^{f(T) S(\alpha)} x(f(T))^{r \alpha} y(f(T))^{r(1-\alpha)} \\
& =F_{r}(x(T)) \oplus_{S} F_{r}(y(T)),
\end{aligned}
$$

where the residue morphism forces $F_{r}(x(T))=x(f(T))^{r}$ for some invertible $f$, depending on $r$. We see from the above that $f(T)=T / r$, proving the claim.

This forces the characters to have the same form as in the Shannon entropy case, i.e., $\chi_{T}(f)(T)=f(T)^{1 / T}$. However, these characters are additive only if $(x(T) \oplus S$ $y(T))^{1 / T}=x^{1 / T}+y^{1 / T}$, which means $S$ must produce the same thermodynamic structure as the Shannon entropy, hence, by a theorem above, $S$ is the Shannon entropy. Note that this analysis holds also in the $q$-deformed Witt construction leading to the Tsallis entropy discussed in $§ 7.1$.

If we pass to the field of fractions of these characters, and consider further infinite sums of these characters, the resulting expressions begin to resemble partition functions in the Euclidean path integral formulation, see $\$ 7$ in [10]. Indeed, if one uses instead $\mathbb{R}^{\min ,+} \cup\{\infty\}$, these are equal to equilibrium free energies of the type observed in $\S 4$. The failure of the additivity of the characters in $\mathcal{R}^{\text {un }}$ can thus be interpreted in terms of nonextensivity. This suggests that, as this candidate for $\mathcal{R}^{\text {un }}$ 
is investigated, new algebraic interpretations of nonextensivity will arise. It would also be interesting to see if a notion of character which is additive on the $q$-deformed Witt construction could give rise to a one-parameter family of $\mathcal{R}^{\text {un 's. }}$

11.4. Thermodynamics in positive characteristics. The main motivation for the Witt construction in characteristic one given in [10] and [11], which provides the prototype example of a thermodynamic semiring built on the Shannon entropy, is to provide an analog in characteristic one of the formulae for the summation of Teichmüller representatives in the case of multiplicative lifts to $\mathbb{Z}_{p}$ of the characteristic $p$ elements in $\mathbb{F}_{p}$.

One can then reverse the point of view and start from the more general thermodynamic semirings associated to other forms of entropy, such as Rényi, Tsallis, Kullback-Leibler, with their axiomatic characterizations, and look for characteristic $p$ analogs of non-extensive thermodynamics and other such variants of statistical physics.

For instance, we saw in $\$ 7$ above that there is a one-parameter deformation of the Witt construction in characteristic one, which yields a characterization of the Tsallis entropy $\mathrm{Ts}_{\alpha}$ as the unique binary information measure that satisfies the associativity, commutativity and unity constraints for this deformed $\oplus_{S, T, \alpha}$ operation.

One thinks of the original $\oplus_{\mathrm{Sh}, T}$ with the Shannon entropy as in [10] and [11], as being the correct analog in characteristic one of the $p$-adic Witt construction

$$
x \oplus_{w} y=\sum_{s \in I_{p}} w_{p}(s) x^{s} y^{1-s},
$$

with $I_{p}$ the set of rational numbers in $[0,1]$ with denominator a power of $p$ and

$$
w_{p}(s)=\sum_{a / p^{n}=s} w\left(p^{n}, a\right) T^{n} \in \mathbb{F}_{p}((T)),
$$

where the $w\left(p^{n}, k\right) \in \mathbb{Z} / p \mathbb{Z}$, for $0<k<p^{n}$ are determined by the addition of Teichmüller representatives

$$
\tau(x)+\tau(y)=\tau(x+y)+\sum_{n=1}^{\infty} \tau\left(\sum w\left(p^{n}, k\right) x^{k / p^{n}} y^{1-k / p^{n}}\right) p^{n} .
$$

Thus, one can equivalently think of the universal sequence of the $w\left(p^{n}, k\right)$ as being the characteristic $p$ analog of the Shannon information. Adopting this viewpoint, one would then expect that the one-parameter deformation of the Witt construction in characteristic one described in \$7, which leads naturally from the Shannon entropy to the non-extensive Tsallis entropy, may correspond to an analogous deformation of the original $p$-adic Witt construction that leads to a notion of non-extensive entropy and non-ergodic thermodynamics in characteristic $p$.

It should be mentioned that there are in fact interesting known $q$-deformations of the Witt constructions, see for instance [40]. These can naturally be described within 
the setting of $\Lambda$-rings (see [40]). This seems especially useful, in view of the whole approach to $\mathbb{F}_{1}$ geometry based on $\Lambda$-rings, developed by James Borger in [7] and [8], [9] (see also [36], [37] for other related viewpoints). However, a reader familiar with the positive characteristic Witt construction will notice that Connes and Consani's construction generalize the $p$-Witt ring from a rather unconventional expression for its addition. This is difficult to translate into the $\Lambda$-ring approach to the Witt ring. A definition of $\Lambda$-rings in characteristic one which reproduces the Witt rings considered in this paper would likely be interesting both geometrically and physically.

This also suggests that identifying suitable analogs of other entropy functions (Tsallis, Rényi, Kullback-Leibler) in characteristic $p$, via deformations of the ring of Witt vectors, may also further our understanding of $\mathbb{F}_{1}$-geometry in the $\Lambda$-ring approach.

Acknowledgment. This paper is based on the results of the second author's summer research project, supported by the Summer Undergraduate Research Fellowship program at Caltech. The first author is partly supported by NSF grants DMS-0901221 and DMS-1007207.

\section{References}

[1] S. Abe and Y. Okamoto (eds.), Nonextensive statistical mechanics and its applications. Lecture Notes in Phys. 560, Springer-Verlag, Berlin 2001. Zbl 0979.00041 MR 1864017

[2] S.-i. Amari, Information geometry on hierarchy of probability distributions. IEEE Trans. Inform. Theory 47 (2001), 1701-1711. Zbl 0997.94009 MR 1842511

[3] S.-i. Amari and H. Nagaoka, Methods of information geometry. Transl. Math. Monographs 191, Amer. Math. Soc., Providence, RI, 2000. Zbl 0960.62005 MR 1800071

[4] J. C. Baez, T. Fritz, and T. Leinster, A characterization of entropy in terms of information loss. Entropy 13 (2011), 1945-1957. MR 2868846

[5] J. Baez, T. Fritz, and T. Leinster, Convex spaces and an operadic approach to entropy. See http://ncatlab.org/johnbaez/show/Entropy+as+a+functor

[6] C. Beck and F. Schlögl, Thermodynamics of chaotic systems. Cambridge Nonlinear Sci. Ser. 4, Cambridge University Press, Cambridge 1993. Zbl 0847.58051 MR 1237638

[7] J. Borger, Lambda-rings and the field with one element. Preprint 2009. arXiv:0906.3146

[8] J. Borger, The basic geometry of Witt vectors, I: The affine case. Algebra Number Theory 5 (2011), 231-285. Zbl 1276.13018 MR 2833791

[9] J. Borger, The basic geometry of Witt vectors, II: Spaces. Math. Ann. 351 (2011), 877-933. Zbl 1251.13019 MR 2854117

[10] A. Connes, The Witt construction in characteristic one and quantization. In Noncommutative geometry and global analysis, Contemp. Math. 546, Amer. Math. Soc., Providence, RI, 2011, 83-113. Zbl 1245.13015 MR 2815131 
[11] A. Connes and C. Consani, From monoids to hyperstructures: in search of an absolute arithmetic. In Casimir force, Casimir operators and the Riemann hypothesis, Walter de Gruyter, Berlin 2010, 147-198. Zbl 1234.14002 MR 2777715

[12] A. Connes, C. Consani, and M. Marcolli, Fun with $\mathbb{F}_{1}$. J. Number Theory 129 (2009), 1532-1561. Zbl 1228.11143 MR 2521492

[13] I. Csiszár, Axiomatic characterization of information measures. Entropy 10 (2008), 261-273. Zbl 1179.94043

[14] E. M. F. Curado and C. Tsallis, Generalized statistical mechanics: connection with thermodynamics. J. Phys. A 24 (1991), L69-L72; Corrigenda: ibid. 24 (1991), 3187, and 25 (1992), 1019. MR 1090024

[15] T. Davis, Catalan numbers. Unpublished notes, November 26, 2006. http://geometer.org/mathcircles/catalan.pdf

[16] K. Denbigh, The principles of chemical equilibrium. 4th ed., Cambridge University Press, Cambridge 1981.

[17] D. K. Faddeev, On the concept of entropy of a finite probabilistic scheme. Uspehi Mat. Nauk (N.S.) 11 (1956), No. 1, 227-231. Zbl 0071.13103 MR 0077814

[18] T. Fritz, A presentation of the category of stochastic matrices. Preprint 2009. arXiv:0902.2554

[19] T. Fritz, Convex spaces I: Definition and examples. Preprint 2009. arXiv:0903.5522

[20] S. Furuichi, On uniqueness theorems for Tsallis entropy and Tsallis relative entropy. IEEE Trans. Inform. Theory 51 (2005), 3638-3645. MR 2237530

[21] M. Gell-Mann and C. Tsallis (eds.), Nonextensive entropy -- interdisciplinary applications. Santa Fe Institute Studies in the Sciences of Complexity, Oxford University Press, Oxford 2004. Zbl 1127.82004 MR 2073730

[22] S. Ikeda, T. Tanaka, and S.-i. Amari, Stochastic reasoning, free energy, and information geometry. Neural Comput. 16 (2004), 1779-1810. Zbl 1090.68106

[23] I. Itenberg and G. Mikhalkin, Geometry in tropical limit. Math. Semesterber. 59 (2012), 57-73. Zbl 1253.14058 MR 2902202

[24] I. Itenberg, G. Mikhalkin, and E. Shustin, Tropical algebraic geometry. 2nd ed., Oberwolfach Seminars 35, Birkhäuser, Basel 2009. Zbl 1165.14002 MR 2508011

[25] M. Kapranov, Thermodynamics and the moment map. Preprint 2011. arXiv:1108.3472

[26] A. I. Khinchin, Mathematical foundations of information theory. Dover Publications, New York 1957. Zbl 0088.10404 MR 0092709

[27] S. Kullback, Information theory and statistics. John Wiley and Sons, Inc., New York; Chapman and Hall, Ltd., London 1959. Zbl 0088.10406 MR 0103557

[28] S. Kullback and R. A. Leibler, On information and sufficiency. Ann. Math. Statistics 22 (1951), 79-86. Zbl 0042.38403 MR 0039968

[29] E. K. Lenzi, R. S. Mendes, and L. R. da Silva, Statistical mechanics based on Renyi entropy. Phys. A 280 (2000), 337-345. MR 1758523

[30] G. L. Litvinov, The Maslov dequantization, and idempotent and tropical mathematics: a brief introduction. Zap. Nauchn. Sem. S.-Peterburg. Otdel. Mat. Inst. Steklov. (POMI) 326 (2005), 145-182; English transl. J. Math. Sci. 140 (2007), 426-444. MR 2183219 
[31] G. L. Litvinov, Tropical mathematics, idempotent analysis, classical mechanics and geometry. In Spectral theory and geometric analysis, Contemp. Math. 535, Amer. Math. Soc., Providence, RI, 2011, 159-186. Zbl 1223.14070 MR 2560756

[32] J. López Peña and O. Lorscheid, Torified varieties and their geometries over $\mathbb{F}_{1}$. Math. Z. 267 (2011), 605-643. Zbl 1220.14021 MR 2776051

[33] F. Lorenz, Algebra, Vol. II: Fields with structure, algebras, and advanced topics. Universitext, Springer-Verlag, New York 2008. Zbl 05209740 MR 2371763

[34] P. Lotito, J.-P. Quadrat, and E. Mancinelli, Traffic assignment \& Gibbs-Maslov semirings. In Idempotent mathematics and mathematical physics, Contemp. Math. 377, Amer. Math. Soc., Providence, RI, 2005, 209-219. Zbl 1176.90107 MR 2149006

[35] D. Maclagan and B. Sturmfels, Introduction to tropical geometry. Preprint 2009. http://homepages.warwick.ac.uk/staff/D.Maclagan/papers/TropicalBook.html

[36] Yu. I. Manin, Cyclotomy and analytic geometry over $\mathbb{F}_{1}$. In Quanta of maths, Clay Math. Proc. 11, Amer. Math. Soc., Providence, RI, 2010, 385-408. Zbl 1231.14018 MR 2732059

[37] M. Marcolli, Cyclotomy and endomotives. p-Adic Numbers Ultrametric Anal. Appl. 1 (2009), 217-263. Zbl 1236.46067 MR 2566053

[38] J. P. May, The geometry of iterated loop spaces. Lectures Notes in Math. 271, SpringerVerlag, Berlin 1972. Zbl 0244.55009 MR 0420610

[39] J. P. May, Operads, algebras and modules. In Operads: Proceedings of Renaissance Conferences (Hartford, CT/Luminy, 1995), Contemp. Math. 202, Amer. Math. Soc., Providence, RI, 1997, 15-31. Zbl 0879.18001 MR 1436914

[40] Y.-T. Oh, q-deformation of Witt-Burnside rings. Math. Z. 257 (2007), 151-191. Zbl 1152.19001 MR 2318574

[41] L. Pachter and B. Sturmfels, Tropical geometry of statistical models. Proc. Natl. Acad. Sci. USA 101 (2004), 16132-16137. Zbl 1135.62302 MR 2114586

[42] Ya. Pesin and V. Climenhaga, Lectures on fractal geometry and dynamical systems. Student Math. Library 52, Amer. Math. Soc., Providence, RI, 2009. Zbl 1186.37003 MR 2560337

[43] J. P. Quadrat and M.-P. W. Group, Min-plus linearity and statistical mechanics. Markov Process. Related Fields 3 (1997), 565-587. Zbl 0905.90174 MR 1607091

[44] J. Rabinoff, The theory of Witt vectors. Unpublished notes, 2007. www.math.harvard.edu/ rabinoff/misc/witt.pdf

[45] A. Rényi, On measures of entropy and information. In Proc. 4th Berkeley Sympos. Math. Statist. and Prob., Vol. I, University California Press, Berkeley, Calif. 1961, 547-561. Zbl 0106.33001 MR 0132570

[46] A. Rényi, On the foundations of information theory. Rev. Inst. Internat. Statist 33 (1965), 1-14. Zbl 0161.16903 MR 0181483

[47] J.-P. Serre, Local fields. Graduate Texts in Math. 67, Springer-Verlag, New York 1979. Zbl 0423.12016 MR 554237

[48] C. Soulé, Les variétés sur le corps à un élément. Moscow Math. J. 4 (2004), 217-244. Zbl 1103.14003 MR 2074990 
[49] H. Suyari, Generalization of Shannon-Khinchin axioms to nonextensive systems and the uniqueness theorem for the nonextensive entropy. IEEE Trans. Inform. Theory $\mathbf{5 0}$ (2004), 1783-1787. MR 2096846

[50] S. Thurner and R. Hanel, What do generalized entropies look like? An axiomatic approach for complex, non-ergodic systems. Preprint 2011. arXiv:1104.2070

[51] J. Tits, Sur les analogues algébriques des groupes semi-simples complexes. In Colloque d'algèbre supérieure, tenu à Bruxelles du 19 au 22 décembre 1956, Centre Belge de Recherches Mathématiques, Établissements Ceuterick, Louvain; Librairie GauthierVillars, Paris 1957, 261-289. Zbl 0084.15902 MR 0108765

[52] C. Tsallis, Possible generalization of Boltzmann-Gibbs statistics. J. Statist. Phys. 52 (1988), 479-487. Zbl 1082.82501 MR 968597

[53] C. Tsallis, Nonextensive statistical mechanics: construction and physical interpretation. In Nonextensive entropy-interdisciplinary applications, St. Fe Inst. Stud. Sci. Complex., Oxford University Press, Oxford 2004, 1-53. Zbl 1127.82004 MR 2074384

[54] O. Viro, Hyperfields for tropical geometry I. Hyperfields and dequantization. Preprint 2010. arXiv:1006.3034

[55] A. Weil, On the Riemann hypothesis in function fields. Proc. Nat. Acad. Sci. USA 27 (1941), 345-347. Zbl 0061.06406 MR 0004242

Received October 5, 2011; revised May 4, 2012

M. Marcolli, Mathematics Department, Mail Code 253-37, Caltech, 1200 E. California Blvd. Pasadena, CA 91125, U.S.A.

E-mail: matilde@caltech.edu

R. Thorngren, Mathematics Department, Mail Code 253-37, Caltech, 1200 E. California Blvd. Pasadena, CA 91125, U.S.A.

E-mail: rthorngr@caltech.edu 